\title{
Global Harmonic Current Rejection of Nonlinear Backstepping Control with Multivariable Adaptive Internal Model Principle for Grid-Connected Inverter under Distorted Grid Voltage
}

\author{
Yang Yu, Zengqiang Mi, Yiming Che, Tong Zhao, and Yikun Xu \\ State Key Laboratory of Alternate Electrical Power System with Renewable Energy Sources, North China Electric Power University, \\ Baoding 071003, China
}

Correspondence should be addressed to Yang Yu; ncepu_yy@163.com

Received 28 May 2013; Accepted 8 July 2013

Academic Editor: Zhiguang Feng

Copyright (C) 2013 Yang Yu et al. This is an open access article distributed under the Creative Commons Attribution License, which permits unrestricted use, distribution, and reproduction in any medium, provided the original work is properly cited.

\begin{abstract}
Based on a brief review on current harmonics generation mechanism for grid-connected inverter under distorted grid voltage, the harmonic disturbances and uncertain items are immersed into the original state-space differential equation of grid-connected inverter. A new algorithm of global current harmonic rejection based on nonlinear backstepping control with multivariable internal model principle is proposed for grid-connected inverter with exogenous disturbances and uncertainties. A type of multivariable internal model for a class of nonlinear harmonic disturbances is constructed. Based on application of backstepping control law of the nominal system, a multivariable adaptive state feedback controller combined with multivariable internal model and adaptive control law is designed to guarantee the closed-loop system globally uniformly bounded, which is proved by a constructed Lyapunov function. The presented algorithm extends rejection of nonlinear single-input systems to multivariable globally defined normal form, the correctness and effectiveness of which are verified by the simulation results.
\end{abstract}

\section{Introduction}

Along with the strong demand of electric power energy, conventional fossil fuels are gradually reduced. The exploiting of renewable energy is an inevitable route for development of sustainable society. According to the planning of EU Commission, the renewable energy goal is achieving $20 \%$ of energy consumption from sustainable sources by 2020 [1].

Renewable energy sources include wind (onshore and offshore), solar PV, hydroenergy, biomass energy, geothermal energy, and tidal energy. Currently, two of main utilization approaches of renewable energy are off-grid power generation and connected to utility grid. In the past decade, the renewable energy sources connected to utility grid have achieved a considerable growth with the forceful needs of electric power energy [2]. Grid-connected inverter, which is one of the key technologies, supports renewable energy to be transmitted to the utility grid. In design, pulse width modulation (PWM) and the corresponding inverter control system require grid voltage as an ideal sine wave. Actually, it is difficult to keep a perfect sine wave of grid voltage under a variety of nonlinear loads and unexpected network failures. Furthermore, the existing researches have shown distorted grid voltage, such as dips [3], asymmetry [4], and harmonics [5], which will have a significantly adverse influence to grid power quality. In other words, rejection of current harmonics for grid-connected inverter under distorted grid voltage is a useful work for the normal operation of power networks [6].

The rejection solutions of current harmonics for gridconnected inverter mainly consist of two categories: hardware implementation and software programming. A simple and practical scheme of hardware implementation is adding filters to inverter's output. The aim of adding filters is to compensate for the fluctuations of grid voltage. In [7], LCL filters are designed for grid-connected converters. However, the application of LCL filters may encounter the resonance problem. Close by the resonant frequency, the impedance of the filters is small, and the harmonic current in corresponding frequency would be amplified, even beyond the harmonic standard [8]. In addition, new hardware will 
certainly increase the investment of the system. One of the rejection ways for software programming is to design a proportional resonant $(\mathrm{PR})$ controller to improve the gain in background harmonic [9]. Nevertheless, with the increasing of the background harmonic frequency, the PR controller can reduce the phase margin of the system [10]. The other one is application of feedforward control of grid voltage to increase output impedance of the system. The feedforward control has no impact on phase margin of the system [11], but the scheme, which only depends on the proportion feedforward to regulation, cannot eliminate the influence of background harmonic [12].

For the disadvantages of the above methods, some scholars attempt to apply modern control theory to improve the capability of harmonics disturbance rejection. The nonlinear control methods, such as repetitive control $[13,14], H_{\infty}$ control [15, 16], prediction control [17-19], backstepping control [20-22], and fuzzy-adaptive control [23-26], can deal with the nonlinearity and uncertainty of nonlinear models better. However, these preexisting rejection control methods have the following problems more or less.

(1) The harmonics rejection algorithms are concerned primarily with the single-input field; the research of multiple-input field is rarely mentioned.

(2) For an operating grid-connected system, the parameters of resistance and inductance for the system are often time varying and cannot be measured accurately. Hence, these proportional-integral-based controllers, which take no account of the time-varying characteristics of the parameters, cannot work very efficiently.

(3) The parameter design of robust controller is relatively complicated and needs large amount of calculations.

In the paper, a multivariable state feedback control algorithm based on nonlinear backstepping control with multivariable internal model principle (MIMP) and adaptive control law (ACL) for grid-connected inverter is proposed to reject the harmonic disturbance produced by a class of exosystem and uncertainties related with system states caused by modeling perturbations, parameter uncertainty or actuator end disturbance. The main contributions in the paper are the following.

(1) A new harmonic rejection algorithm based on nonlinear backstepping control with MIMP and ACL is proposed in the paper, which extends the disturbance rejection of the nonlinear single-input system to multivariable globally defined normal form in contrast with the previous literatures.

(2) A new type of nonlinear multivariable internal model for a class of nonlinear harmonic disturbances and uncertain items related with system states is constructed.

(3) The proposed algorithm is practically applied to reject current harmonic disturbances for grid-connected inverter under distorted grid voltage.

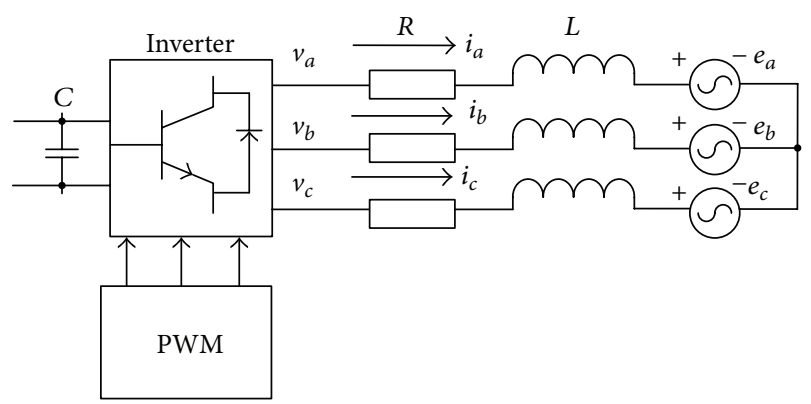

FIGURE 1: Modeling of grid-connected inverter.

The paper is organized as the following: it starts with an introduction to the research status of current harmonics rejection for grid-connected inverter in Section 1. The modeling of state differential equation for grid-connected inverter is constructed and an analysis of current harmonics generation mechanism is given in Section 2. In addition, Section 2 describes the control and mathematical problem concerned in the paper. Nonlinear multivariable internal model is presented and designed in Section 3. In Section 4, nonlinear multivariable adaptive state feedback controller is proposed and the global robust stabilization is demonstrated. Section 5 gives the results of numerical simulation. Finally, the conclusions are summarized in Section 6.

\section{Problem Formulation}

2.1. Modeling of Grid-Connected Inverter. The model of gridconnected inverter can be shown in Figure 1. In Figure 1, $R$ and $L$ represent the equivalent series resistance and inductor, respectively, $C$ is the capacitance, $v_{a b c}$ and $i_{a b c}$ are three-phase output voltage and grid current of three-phase inverter, and $e_{a b c}$ represents three-phase grid voltage.

In terms of Figure 1, the voltage equations of three-phase grid-connected inverter in stationary $a b c$ reference frame can be written as

$$
\begin{aligned}
& v_{a}=R i_{a}+L \frac{d i_{a}}{d t}+e_{a}, \\
& v_{b}=R i_{b}+L \frac{d i_{b}}{d t}+e_{b}, \\
& v_{c}=R i_{c}+L \frac{d i_{c}}{d t}+e_{c} .
\end{aligned}
$$

Application of the park transformation to (1) in rotating $d q$ reference frame equation (1) is converted into

$$
\begin{aligned}
& v_{d}=R i_{d}+L \frac{d i_{d}}{d t}-\omega L i_{q}+e_{d}, \\
& v_{q}=R i_{q}+L \frac{d i_{q}}{d t}+\omega L i_{d}+e_{q} ;
\end{aligned}
$$


namely,

$$
\left[\begin{array}{l}
\frac{d i_{d}}{d t} \\
\frac{d i_{q}}{d t}
\end{array}\right]=\left[\begin{array}{cc}
-\frac{R}{L} & \omega \\
-\omega & -\frac{R}{L}
\end{array}\right]\left[\begin{array}{l}
i_{d} \\
i_{q}
\end{array}\right]+\left[\begin{array}{cc}
\frac{1}{L} & 0 \\
0 & \frac{1}{L}
\end{array}\right]\left[\begin{array}{cc}
v_{d} & -e_{d} \\
v_{q} & -e_{q}
\end{array}\right]
$$

where $v_{d q}$ and $i_{d q}$ represent the voltage and current of gridconnected inverter in rotating $d q$ reference frame, $e_{d q}$ is the grid voltage in $d q$ reference frame, and $\omega$ is the synchronous angular velocity of the grid.

Rearrange (2) in the form of state space as follows:

$$
\begin{gathered}
\dot{\mathbf{x}}=\mathbf{A} \mathbf{x}+\mathbf{B}(\mathbf{v}-\mathbf{e}), \\
\mathbf{y}=\mathbf{C x},
\end{gathered}
$$

where $\mathbf{x}, \mathbf{v}$, and $\mathbf{y}$ are the state vector, the control input vector, and the output vector, respectively, $\mathbf{e}$ is the voltage vector of the grid, $\mathbf{x}=\left[\begin{array}{ll}i_{d} & i_{q}\end{array}\right]^{T}, \mathbf{v}=\left[\begin{array}{ll}v_{d} & v_{q}\end{array}\right]^{T}, \mathbf{y}=\mathbf{x}, \mathbf{e}=$ $\left[\begin{array}{ll}e_{d} & e_{q}\end{array}\right]^{T}, \mathbf{A}=\left[\begin{array}{cc}-R / L & \omega \\ -\omega & -R / L\end{array}\right], \mathbf{B}=\left[\begin{array}{cc}1 / L & 0 \\ 0 & 1 / L\end{array}\right]$, and $\mathbf{C}=\left[\begin{array}{ll}1 & 0 \\ 0 & 1\end{array}\right]$.

2.2. Current Harmonics Generation Mechanism for GridConnected Inverter. In terms of Figure 1, the harmonic voltage equations of three-phase grid-connected inverter instationary $a b c$ reference frame can be written as

$$
\mathbf{v}_{a b c k}=R \mathbf{i}_{a b c k}+L \frac{d \mathbf{i}_{a b c k}}{d t}+\mathbf{e}_{a b c k},
$$

where $k$ is the harmonic order, $\mathbf{v}_{a b c k}$ is the control voltage vector, $\mathbf{i}_{a b c k}$ is the current vector of harmonics, and $\mathbf{e}_{a b c k}$ is the unbalanced harmonic voltage vector of the grid.

Assume that the distorted voltage of the network contains the 5 th and 7 th harmonics. In stationary $a b c$ reference frame, the rotating direction of the 5th harmonics is contrary to fundamental harmonics, and its rotating electrical angular velocity is $-5 \omega$. However, the rotating direction of the 7 th harmonics is the same as the fundamental harmonics, and its rotating electrical angular velocity is $7 \omega$. Indeed, the distorted voltage of the network may include more harmonics in addition to the 5th and 7th harmonics, such as the $3 \mathrm{rd}$, 11 th, and 13th. The rotating direction of the 11th harmonics is exactly identical to the 5th, except that the rotating electrical angular velocity is $-11 \omega$. A similar relationship exists in the 7 th and 13th harmonics. In order to reveal the essence of the problem more simply, consider only the 5th and 7 th harmonics and construct multiple synchronous rotating reference frames shown in Figure 2. For convenience, in what follows, +/- in superscript represents $+/$ - rotating direction, respectively; 5 and 7 in superscript describe the rotating direction of the 5 th and 7 th harmonics, respectively; 1,5 , and 7 in subscript demonstrate the 1st, 5 th, and 7 th harmonics, respectively.

In rotating $d q$ reference frame of the 5 th harmonics, the voltage equation of the 5 th harmonics can be written as

$$
\mathbf{v}_{d q 5}=R \mathbf{i}_{d q 5}+L \frac{d \mathbf{i}_{d q 5}}{d t}+\mathbf{e}_{d q 5}-j 5 \omega L i_{d q 5},
$$

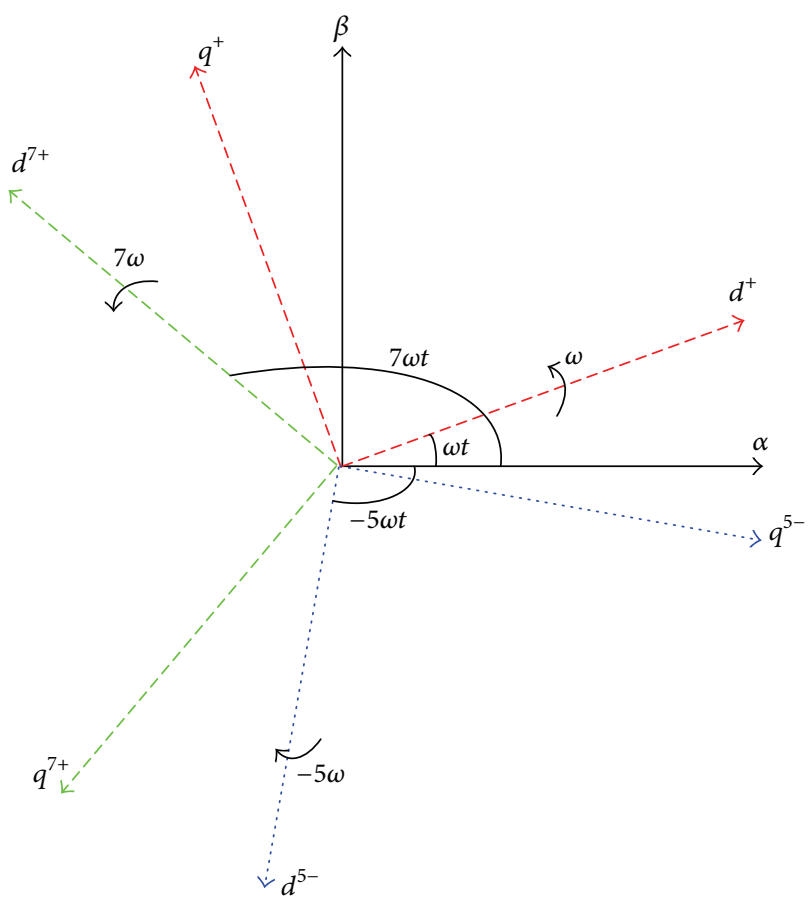

FIGURE 2: Multiple synchronous rotating reference frames.

where

$$
\begin{aligned}
& \mathbf{v}_{d q 5}=\mathbf{v}_{d q 1}^{+} e^{j 6 \omega t}+\mathbf{v}_{d q 5}^{5-}+\mathbf{v}_{d q 7}^{7+} e^{j 12 \omega t} \\
& \mathbf{i}_{d q 7}=\mathbf{i}_{d q 1}^{+} e^{j 6 \omega t}+\mathbf{i}_{d q 5}^{5-}+\mathbf{i}_{d q 7}^{7+} e^{j 12 \omega t} \\
& \mathbf{e}_{d q 5}=\mathbf{e}_{d q 1}^{+} e^{j 6 \omega t}+\mathbf{e}_{d q 5}^{5-}+\mathbf{e}_{d q 7}^{7+} e^{j 12 \omega t}
\end{aligned}
$$

Equation (7) indicates that only voltage and current components of the 5 th harmonics are DC signals in rotating $d q$ reference frame of the 5th harmonics. Voltage and current components of the remaining harmonics are AC signals. One other thing to note is that all of the harmonic components in rotating $d q$ reference frame of fundamental wave are AC signals.

In a similar way, the voltage equation of the 7 th harmonics can be written as

$$
\mathbf{v}_{d q 7}=R \mathbf{i}_{d q 7}+L \frac{d \mathbf{i}_{d q 7}}{d t}+\mathbf{e}_{d q 7}+j 7 \omega L i_{d q 7} .
$$

Equations (6) and (8) demonstrate that once the grid voltage becomes unbalance, the controller designed by the principle of ideal sine wave will not be able to compensate for harmonic voltage, and the current waveform of the grid will be distorted.

2.3. Control Problem Description. Use $\mathbf{D}(\mathbf{w})$ and $\boldsymbol{\delta}(\mathbf{x}, t)$ to describe the exogenous disturbance of voltage harmonics and the uncertain items related with system states caused by modeling perturbations, parameter uncertainties, or actuator 
end disturbances, respectively, then the state equation in (4) can be rewritten as a multivariable system as follows:

$$
\begin{aligned}
\dot{\mathbf{x}} & =\mathbf{A} \mathbf{x}+\mathbf{B}\left(\mathbf{v}-\mathbf{e}_{0}-\mathbf{D}(\mathbf{w})+\boldsymbol{\delta}(\mathbf{x}, t)\right) \\
& =\mathbf{f}(\mathbf{x})+\sum_{i=1}^{2} \mathbf{g}_{i}(\mathbf{x})\left(v_{i}-e_{i 0}-D_{i}(\mathbf{w})+\delta_{i}(\mathbf{x}, t)\right)
\end{aligned}
$$

$$
1 \leq i \leq 2
$$

where $\mathbf{x}=\left[x_{1}, x_{2}\right]^{T}=\left[i_{d}, i_{q}\right]^{T}, \mathbf{f}(\mathbf{x})=\left[\begin{array}{l}-(R / L) x_{1}+\omega x_{2} \\ -\omega x_{1}-(R / L) x_{2}\end{array}\right]=$ $\left[\begin{array}{c}-(R / L) i_{d}+\omega i_{q} \\ -\omega i_{d}-(R / L) i_{q}\end{array}\right], \mathbf{g}_{1}(\mathbf{x})=\mathbf{g}_{2}(\mathbf{x})=1 / L, \mathbf{v}=\left[v_{1}, v_{2}\right]^{T}=$ $\left[v_{d}, v_{q}\right]^{T},\left[e_{10}, e_{20}\right]=\left[e_{d 0}, e_{q 0}\right]$, and $e_{d 0}$ and $e_{q 0}$ are network voltages of fundamental wave in $d q$ reference frame. For a specific network, $e_{d 0}$ and $e_{q 0}$ can be determined easily. The uncertain item $\boldsymbol{\delta}(\mathbf{x}, t)$ in the control input channel satisfies the matching condition. In the paper, the range of variable $i$ is $(1,2)$. For convenience and conciseness, in what follows, the range of variable $i$ is no longer written.

Assumption 1. $\mathbf{w} \in \mathbf{R}^{q}$ is an exogenous signal generated by the following exosystem:

$$
\dot{\mathbf{w}}=\mathbf{W}_{i} \mathbf{w}
$$

where $\mathbf{W}_{i}$ is a pending matrix depending on the exogenous signal.

Assumption 2. Considering disturbance-free and no modeling perturbations, the nominal system of (9) can be written as:

$$
\dot{\mathbf{x}}=\mathbf{f}(\mathbf{x})+\sum_{i=1}^{2} \mathbf{g}_{i}(\mathbf{x})\left(v_{i}-e_{i 0}\right)
$$

there exists a control law of state feedback a follows:

$$
v_{i}^{\prime}=v_{i}-e_{i 0}=\alpha_{i}(\mathbf{x}),
$$

let (11) be asymptotically stable. In addition, there exists a Lyapunov function $V(\mathbf{x})$ which satisfies:

$$
\begin{gathered}
\underline{d}(\|\mathbf{x}\|) \leq V(\mathbf{x}) \leq \bar{d}(\|\mathbf{x}\|), \\
\frac{\partial V(\mathbf{x})}{\partial \mathbf{x}}\left(\mathbf{f}(\mathbf{x})+\sum_{i=1}^{2} \mathbf{g}_{i}(\mathbf{x}) \alpha_{i}(\mathbf{x})\right) \leq-d_{0}(\|\mathbf{x}\|), \\
\left|\frac{\partial V(\mathbf{x})}{\partial \mathbf{x}} \sum_{i=1}^{2} \mathbf{g}_{i}(\mathbf{x})\right|^{2} \leq d_{0}(\|\mathbf{x}\|)
\end{gathered}
$$

where $\underline{d}, \bar{d}$, and $d_{0}$ are all of class $K_{\infty}$ functions.

Assumption 3. There exist smooth functions $r_{i}(x): \mathbf{R}^{n} \rightarrow$ $\mathbf{R}^{q}$ which make

$$
\frac{\partial r_{i}(\mathbf{x})}{\partial \mathbf{x}} \mathbf{g}_{i}(\mathbf{x})=\mathbf{K}_{i}
$$

where $\mathbf{K}_{i}$ are nonzero constant vectors defined in $\mathbf{R}^{q}$.
Assumption 4. There exist a constant $m_{i}$ and a known smooth bounded function $p_{i}(\mathbf{x})$ making the uncertain item $\boldsymbol{\delta}(\mathbf{x}, t)$ satisfy

$$
\left|\delta_{i}(\mathbf{x}, t)\right| \leq m_{i} p_{i}(\mathbf{x})
$$

Remark 5. Similar to the uncertain item $\boldsymbol{\delta}(\mathbf{x}, t)$, the disturbance $\mathbf{D}(\mathbf{w})$ also satisfies the matching condition and can be injected in the input path. Nevertheless, a method of iterative design, such as adaptive backstepping, can extend the presented algorithm to more general instance of strict feedback. Therefore, the matching condition is not critical, and application of the assumption is reasonable.

Remark 6. One of the research points in the paper is stability problem for multivariable input system. Currently, some literatures focus on the point of stability problem, but the focused attention of these literatures is the field of single input and single output [27] or semiglobal stability [28]. For the multivariable system, the research of stability problem will be more challenging, and the core is to transform the stability problem from multivariable system to multiple single-input system [29].

Remark 7. For Assumption 2, (13) and (14) automatically hold when the closed-loop system is asymptotically stable. Equation (15) holds when the closed-loop system is exponentially stable. Nevertheless, Assumption 2 is not a sufficient condition to make the closed-loop system exponentially stable [30].

Remark 8. For Assumption 3, if $\mathbf{g}_{i}(\mathbf{x})$ is a nonzero constant vector, it will be easy to find a solution of $\left(\partial r_{i}(\mathbf{x}) / \partial \mathbf{x}\right) \mathbf{g}_{i}(\mathbf{x})=$ $\mathbf{K}_{i}$ for a non-zero constant vector $\mathbf{K}_{i}$. For a nonconstant vector $\mathbf{g}_{i}(\mathbf{x})$, the solutions can be found more complex with the help of geometric tool [31].

Remark 9. Assumption 4 is to guarantee the boundedness of the uncertain item $\boldsymbol{\delta}(\mathbf{x}, t)$ relative to the system state vector $\mathbf{x}$. For instance, if $\delta_{1}(\mathbf{x}, t)=x_{1} \sin \left(x_{1} t\right), m_{1}$ and $p_{1}(\mathbf{x})$ can be chosen as 1 and $x_{1}$, respectively.

The problem solved in the paper can be described by the following theorem.

Definition 10. For any given compact set $\mathbf{D}_{\mathbf{w}} \in \mathbf{R}^{q}$, state feedback controller $v_{i}$ can always be found to ensure the solution of closed-loop system (9) existing under arbitrary initial conditions for all $\mathbf{w}(\mathbf{0}) \in \mathbf{D}_{\mathbf{w}}$ and $t \geq 0$ and to reject exogenous disturbances and uncertain items.

\section{Design of Nonlinear Multivariable Internal Model}

Application of internal model principle (IMP) to reject the exogenous disturbance $\mathbf{D}(\mathbf{w})$ and uncertain item $\boldsymbol{\delta}(\mathbf{x}, t)$ in the paper is chosen as an indirect method. In other words, an appropriate equation of internal model should be constructed to estimate the nonlinear disturbances and uncertain items. 
The nonlinear equation of exogenous disturbance internal model is designed as

$$
\begin{gathered}
\widehat{D}_{i}(\mathbf{w})=\mathbf{V}_{i} \widehat{\mathbf{w}}_{i}(t), \\
\widehat{\mathbf{w}}_{i}(t)=\widehat{\boldsymbol{\eta}}_{i}(t)+\mathbf{r}_{i}(\mathbf{x}), \\
\dot{\hat{\boldsymbol{\eta}}}_{i}(t)=\left(\mathbf{W}_{i}-\mathbf{K}_{i} \mathbf{V}_{i}\right)\left(\widehat{\boldsymbol{\eta}}_{i}(t)+\mathbf{r}_{i}(\mathbf{x})\right) \\
-\mathbf{K}_{i} v_{i}^{\prime}(\mathbf{x})-\frac{\partial \mathbf{r}_{i}(\mathbf{x})}{\partial \mathbf{x}} \mathbf{f}(\mathbf{x}),
\end{gathered}
$$

where $\widehat{\mathbf{w}}(t)$ is the estimation value of exogenous signal $\mathbf{w}(t), \widehat{\boldsymbol{\eta}}_{i}$ is the introduced auxiliary vector, $\widehat{\boldsymbol{\eta}}_{i}=$ $\left[\begin{array}{ll}\widehat{\eta}_{i 1} & \widehat{\eta}_{i 2} \cdots \widehat{\eta}_{i p}\end{array}\right], \mathbf{r}_{i}(\mathbf{x})$ is a vector constructed by the smooth functions $r_{i}(\mathbf{x}), \mathbf{r}_{i}(\mathbf{x})=\left[\begin{array}{ll}r_{i}(\mathbf{x}) & 0 \cdots 0\end{array}\right]_{1 \times p}$, and the matrix $\mathbf{K}_{i} \in \mathbf{R}^{q}$ is selected satisfying Assumption 3 and makes the matrix $\left(\mathbf{W}_{i}-\mathbf{K}_{i} \mathbf{V}_{i}\right)$ be Hurwitz; that is, positive definite matrices $\mathbf{P}_{i}$ and $\mathbf{Q}_{i}$ are always existing and satisfy

$$
\mathbf{P}_{i}\left(\mathbf{W}_{i}-\mathbf{K}_{i} \mathbf{V}_{i}\right)+\left(\mathbf{W}_{i}-\mathbf{K}_{i} \mathbf{V}_{i}\right)^{T} \mathbf{P}_{i}=-\mathbf{Q}_{i} .
$$

In terms of Assumption 4, the adaptive internal model of uncertain item is devised as

$$
\widehat{\delta}_{i}(\mathbf{x}, t)=m_{i} \mathbf{K}_{i} p_{i}(\mathbf{x}) \tanh \left(\frac{\widehat{\eta}_{i}^{T} \mathbf{P}_{i}^{T} \mathbf{K}_{i} p_{i}(\mathbf{x})}{\lambda_{i}}\right),
$$

where $m_{i}$ and $p_{i}(\mathbf{x})$ indicate a constant and a known smooth function defined in Assumption 4, respectively, and $\lambda_{i}$ is a designed constant.

Define an auxiliary error vector as follows:

$$
e r_{i}(t)=\mathbf{w}_{i}(t)-\widehat{\mathbf{w}}_{i}(t) .
$$

With the derivative of (23) along with (9), (10) and (19), we can obtain

$$
\begin{aligned}
\dot{e r}_{i}(t)= & \dot{\mathbf{w}}_{i}(t)-\dot{\widehat{\mathbf{w}}}_{i}(t)=\mathbf{W}_{i} \mathbf{w}_{i}-\dot{\overrightarrow{\boldsymbol{\eta}}}_{i}(t)-\frac{\partial \mathbf{r}_{i}(\mathbf{x})}{\partial \mathbf{x}} \frac{\partial \mathbf{x}}{\partial t} \\
= & \mathbf{W}_{i} \mathbf{w}_{i}-\left(\mathbf{W}_{i}-\mathbf{K}_{i} \mathbf{V}_{i}\right)\left(\widehat{\boldsymbol{\eta}}_{i}(t)+\mathbf{r}_{i}(\mathbf{x})\right) \\
& +\mathbf{K}_{i} \mathbf{v}_{i}^{\prime}(\mathbf{x})+\frac{\partial \mathbf{r}_{i}(\mathbf{x})}{\partial x} \mathbf{f}(\mathbf{x})-\frac{\partial \mathbf{r}_{i}(\mathbf{x})}{\partial x} \\
& \quad \times\left(\mathbf{f}(\mathbf{x})+\mathbf{g}_{i}(\mathbf{x})\left(v_{i}-e_{i 0}-D_{i}(\mathbf{w})+\delta_{i}(\mathbf{x}, t)\right)\right) \\
= & \mathbf{W}_{i} \mathbf{w}_{i}-\left(\mathbf{W}_{i}-\mathbf{K}_{i} \mathbf{V}_{i}\right)\left(\widehat{\boldsymbol{\eta}}_{i}(t)+\mathbf{r}_{i}(\mathbf{x})\right)+\mathbf{K}_{i} v_{i}^{\prime}(x) \\
& -\frac{\partial \mathbf{r}_{i}(\mathbf{x})}{\partial x} \mathbf{g}_{i}(\mathbf{x})\left(v_{i}-e_{i 0}-D_{i}(\mathbf{w})+\delta_{i}(\mathbf{x}, t)\right) \\
= & \mathbf{W}_{i} \mathbf{w}_{i}-\left(\mathbf{W}_{i}-\mathbf{K}_{i} \mathbf{V}_{i}\right)\left(\widehat{\boldsymbol{\eta}}_{i}(t)+\mathbf{r}_{i}(\mathbf{x})\right)+\mathbf{K}_{i} v_{i}^{\prime}(\mathbf{x}) \\
& -\mathbf{K}_{i}\left(v_{i}-e_{i 0}-D_{i}(\mathbf{w})+\delta_{i}(\mathbf{x}, t)\right) \\
= & \mathbf{W}_{i} \mathbf{w}_{i}-\left(\mathbf{W}_{i}-\mathbf{K}_{i} \mathbf{V}_{i}\right) \widehat{\mathbf{w}}_{i}(t)+\mathbf{K}_{i} v_{i}^{\prime}(\mathbf{x}) \\
& -\mathbf{K}_{i}\left(v_{i}-e_{i 0}-\mathbf{V}_{i} \mathbf{w}(t)+\delta_{i}(\mathbf{x}, t)\right) \\
= & \mathbf{W}_{i} \mathbf{w}_{i}-\left(\mathbf{W}_{i}-\mathbf{K}_{i} \mathbf{V}_{i}\right) \widehat{\mathbf{w}}(t)+\mathbf{K}_{i} V_{i} \mathbf{w}(t)-\mathbf{K}_{i} \delta_{i}(\mathbf{x}, t) \\
= & \left(\mathbf{W}_{i}-\mathbf{K}_{i} \mathbf{V}_{i}\right) e r_{i}(t)-\mathbf{K}_{i} \delta_{i}(\mathbf{x}, t)
\end{aligned}
$$

\section{Design of Nonlinear State Feedback Controller}

In comparison with the nominal system (11), the original system (9) adds two items: exogenous disturbance $\mathbf{D}(\mathbf{w})$ and uncertain items $\boldsymbol{\delta}(\mathbf{x}, t)$. On the basis of control law of the nominal system, nonlinear state feedback controller of the original system (9) should add two items to compensate for $\mathbf{D}(\mathbf{w})$ and $\boldsymbol{\delta}(\mathbf{x}, t)$. In terms of the idea, nonlinear state feedback controller of the original system (9) is designed as

$$
v_{i}^{\prime}=\alpha_{i}(\mathbf{x})-\widehat{D}_{i}(\mathbf{w})+\theta_{i}(\cdot),
$$

where $\theta(\cdot)$ is a pending design function to compensate for an uncertain item such as modeling perturbation.

Apparently, designing of nonlinear internal model $\widehat{D}_{i}(\mathbf{w})$ is accomplished in Section 3. In what follows, we complete the design of $v_{i}^{\prime}$ in two steps. The first step is application of backstepping theory to devise $\alpha_{i}(\mathbf{x})$, and the second is to complete an adaptive solution of $\theta(\cdot)$ to reject uncertain item $\boldsymbol{\delta}(\mathbf{x}, t)$.

(1) Designing of $\alpha_{i}(\mathbf{x})$. It is assumed that the parameters of grid-connected inverter are known and invariant. The control target of designing $\alpha_{i}(\mathbf{x})$ is to regulate $i_{d}\left(x_{1}\right)$ and $i_{q}\left(x_{2}\right)$ to track the respective reference value $x_{1 \text { ref }}$ and $x_{2 \text { ref }}$.

Firstly, we define the errors as follows:

$$
\begin{aligned}
& e z_{1}=x_{1}-x_{1 \mathrm{ref}}, \\
& e z_{2}=x_{2}-x_{2 \mathrm{ref}} ;
\end{aligned}
$$

the dynamics derived from (26), we can get with

$$
\begin{aligned}
& \dot{e} z_{1}=\dot{x}_{1}-\dot{x}_{1 \mathrm{ref}}, \\
& \dot{e} z_{2}=\dot{x}_{2}-\dot{x}_{2 \mathrm{ref}} .
\end{aligned}
$$

Substituting (9) into (27), we obtain

$$
\begin{aligned}
& \dot{e} z_{1}=\dot{x}_{1}-\dot{x}_{1 \mathrm{ref}}=-\frac{R}{L} x_{1}+\omega x_{2}+\frac{1}{L} \alpha_{1}(\mathbf{x})-\dot{x}_{1 \mathrm{ref}}, \\
& \dot{e} z_{2}=\dot{x}_{2}-\dot{x}_{2 \mathrm{ref}}=-\frac{R}{L} x_{2}-\omega x_{1}+\frac{1}{L} \alpha_{2}(\mathbf{x})-\dot{x}_{2 \mathrm{ref}} .
\end{aligned}
$$

Define a quadratic function as the following:

$$
Q_{1}=\frac{1}{2} c_{1} e z_{1}^{2}
$$

where $c_{1}$ is a positive real number.

Derivative of $Q_{1}$ along (28) can be written as

$$
\dot{Q}_{1}=c_{1} e z_{1} e z_{1}=c_{1} e z_{1}\left(-\frac{R}{L} x_{1}+\omega x_{2}+\frac{1}{L} \alpha_{1}(\mathbf{x})-\dot{x}_{1 \mathrm{ref}}\right) \text {. }
$$

Assume that

$$
-\frac{R}{L} x_{1}+\omega x_{2}+\frac{1}{L} \alpha_{1}(\mathbf{x})-\dot{x}_{1 \mathrm{ref}}=-\frac{e z_{1}}{c_{1}} .
$$


That is,

$$
\alpha_{1}(\mathbf{x})=-\frac{L e z_{1}}{c_{1}}+R x_{1}-L \omega x_{2}+L \dot{x}_{1 \mathrm{ref}} .
$$

Replacing (33) into (31), the derivative of $Q_{1}$ is given by

$$
\dot{Q}_{1}=c_{1} e z_{1} e z_{1}=-e z_{1}^{2} \leq 0 .
$$

Define another quadratic function as the following:

$$
Q_{2}=\frac{1}{2} c_{2} e z_{2}^{2}
$$

where $c_{2}$ is also a positive real number.

Derivative of $Q_{2}$ along (29) is given by

$$
\dot{Q}_{2}=c_{2} e z_{2} e z_{2}=c_{2} z_{2}\left(-\frac{R}{L} x_{2}-\omega x_{1}+\frac{1}{L} \alpha_{2}(x)-\dot{x}_{2 \mathrm{ref}}\right) \text {. }
$$

Suppose that

$$
-\frac{R}{L} x_{2}-\omega x_{1}+\frac{1}{L} \alpha_{2}(x)-\dot{x}_{2 \mathrm{ref}}=-\frac{e z_{2}}{c_{2}} ;
$$

that is,

$$
\alpha_{2}(\mathbf{x})=-\frac{L e z_{2}}{c_{2}}+R x_{2}+L \omega x_{1}+L \dot{x}_{2 \mathrm{ref}} .
$$

Replacing (38) into (36), the derivative of $Q_{2}$ can be written as

$$
\dot{Q}_{2}=c_{2} e z_{2} e z_{2}=-e z_{2}^{2} \leq 0 .
$$

Now, from (30) and (35), the Lyapunov function $V(\mathbf{x})$ defined in Assumption 2 can be written as

$$
V(\mathbf{x})=Q_{1}+Q_{2}=\frac{1}{2} c_{1} e z_{1}^{2}+\frac{1}{2} c_{2} e z_{2}^{2} .
$$

Hence, from (34) and (39), we can see that the controllers $\alpha_{1}(\mathbf{x})$ shown in (33) and $\alpha_{2}(\mathbf{x})$ shown in (38) can stabilize the disturbance-free closed-loop system.

(2) Designing of $\theta(\cdot)$. Under the actual operating environments, the parameters of grid-connected inverter are not always known and invariant. For instance, the inductance $L$ varies with the environment temperature, and the resistance $R$ changes nonlinearly with heating. Hence, the parametric uncertainties in the process of system modeling should be considered to reflect the real condition of the operating system. Due to the uncertainty of modeling perturbations, $\theta(\cdot)$ should be an adaptive controller to reject the perturbations. In terms of the internal model of uncertain items shown in (22), the controller is devised as

$$
\theta_{i}(\mathbf{x}, t)=m_{i} p_{i}(\mathbf{x}) \tanh \left(\frac{(\partial V(\mathbf{x}) / \partial x) g_{i}(\mathbf{x}) p_{i}(\mathbf{x})}{\xi_{i}}\right),
$$

where $\xi_{i}$ is the designed constant.

Convergence Proof of the Proposed Controller. For a start, we give a lemma as the following.
Lemma 11. For any positive number $\varepsilon>0$, there exists $a$ smooth function $h$ making the inequality $|x| \leq x h(x)+\varepsilon$, for all $x \in R$ hold, and $f(0)=0$.

Remark 12. If we choose $h(x)=(1 / 4 \varepsilon) x$, for all $x \in R$, the above inequality holds obviously. In [32], another function meeting the above requirements is given as $h(x)=$ $\tanh (\beta x / \varepsilon)$, for all $x \in R$, where $\beta=e^{-(\beta+1)}$ and $\beta>0$, hence $\beta<1 / 2$.

In terms of (23) and (40), construct a new Lyapunov function as

$$
W=V(\mathbf{x})+\sum_{i=1}^{2} e r_{i}^{T} P_{i} e r_{i} .
$$

With the derivative of (42) along the system (9), (24), and (25), we obtain

$$
\begin{aligned}
& \dot{W}=\frac{\partial V(\mathbf{x})}{\partial \mathbf{x}}\left(\mathbf{f}(\mathbf{x})+\sum_{i=1}^{2} \mathbf{g}_{i}(\mathbf{x})\left(v_{i}-e_{i 0}-D_{i}(\mathbf{w})+\delta_{i}(\mathbf{x}, t)\right)\right) \\
& +\sum_{i=1}^{2}\left(e r_{i}^{T}\left(\mathbf{P}_{i}\left(\mathbf{W}_{i}-\mathbf{K}_{i} \mathbf{V}_{i}\right)+\left(\mathbf{W}_{i}-\mathbf{K}_{i} \mathbf{V}_{i}\right)^{T} \mathbf{P}_{i}\right) e r_{i}\right) \\
& +2 \sum_{i=1}^{2}\left(\operatorname{er}_{i}^{T} \mathbf{P}_{i}\left(-\mathbf{K}_{i} \delta_{i}(\mathbf{x}, t)\right)\right) \\
& =\frac{\partial V(\mathbf{x})}{\partial \mathbf{x}}\left(\mathbf{f}(\mathbf{x})+\sum_{i=1}^{2} \mathbf{g}_{i}(\mathbf{x}) \alpha_{i}(\mathbf{x})\right) \\
& +\frac{\partial V(\mathbf{x})}{\partial \mathbf{x}} \sum_{i=1}^{2} \mathbf{g}_{i}(\mathbf{x})\left(\widehat{D}_{i}(\mathbf{w})-D_{i}(\mathbf{w})\right) \\
& -\frac{\partial V(\mathbf{x})}{\partial \mathbf{x}} \sum_{i=1}^{2} \mathbf{g}_{i}(\mathbf{x})\left(\theta_{i}(\mathbf{x}, t)-\delta_{i}(\mathbf{x}, t)\right) \\
& -\sum_{i=1}^{m}\left(\operatorname{er}_{i}^{T} \mathbf{Q}_{i} e r_{i}\right)+2 \sum_{i=1}^{2}\left(\operatorname{er}_{i}^{T} \mathbf{P}_{i}\left(-\mathbf{K}_{i} \delta_{i}(\mathbf{x}, t)\right)\right) \\
& =\frac{\partial V(\mathbf{x})}{\partial \mathbf{x}}\left(\mathbf{f}(\mathbf{x})+\sum_{i=1}^{2} \mathbf{g}_{i}(\mathbf{x}) \alpha_{i}(\mathbf{x})\right) \\
& +\frac{\partial V(\mathbf{x})}{\partial \mathbf{x}} \sum_{i=1}^{2} \mathbf{g}_{i}(\mathbf{x})\left(\mathbf{V}_{i} \widehat{\mathbf{w}}(t)-V_{i} \mathbf{w}(t)\right) \\
& -\frac{\partial V(\mathbf{x})}{\partial \mathbf{x}} \sum_{i=1}^{2} \mathbf{g}_{i}(\mathbf{x})\left(m_{i} p_{i}(\mathbf{x}) \tanh \right. \\
& \times\left(\frac{(\partial V(\mathbf{x}) / \partial \mathbf{x}) \mathbf{g}_{i}(\mathbf{x}) p_{i}(\mathbf{x})}{\xi_{i}}\right) \\
& \left.-\delta_{i}(\mathbf{x}, t)\right) \\
& -\sum_{i=1}^{m}\left(e r_{i}^{T} \mathbf{Q}_{i} e r_{i}\right)-2 \sum_{i=1}^{2}\left(e r_{i}^{T} \mathbf{P}_{i}\left(\mathbf{K}_{i} \delta_{i}(\mathbf{x}, t)\right)\right) .
\end{aligned}
$$


In terms of Assumption 4, (43) can be rewritten as

$$
\begin{aligned}
\dot{W} \leq & \frac{\partial V(\mathbf{x})}{\partial \mathbf{x}}\left(\mathbf{f}(\mathbf{x})+\sum_{i=1}^{2} \mathbf{g}_{i}(\mathbf{x}) \alpha_{i}(\mathbf{x})\right)+\frac{\partial V(\mathbf{x})}{\partial \mathbf{x}} \sum_{i=1}^{2} \mathbf{g}_{i}(\mathbf{x})\left(\mathbf{V}_{i} e r_{i}(t)\right) \\
& -\frac{\partial V(\mathbf{x})}{\partial \mathbf{x}} \sum_{i=1}^{2} \mathbf{g}_{i}(\mathbf{x})\left(m_{i} p_{i}(\mathbf{x}) \tanh \left(\frac{(\partial V(\mathbf{x}) / \partial \mathbf{x}) \mathbf{g}_{i}(\mathbf{x}) p_{i}(\mathbf{x})}{\xi_{i}}\right)-\left|m_{i} p_{i}(\mathbf{x})\right|\right) \\
& -\sum_{i=1}^{m}\left(e r_{i}^{T} \mathbf{Q}_{i} e r_{i}\right)-2 \sum_{i=1}^{2}\left(e r_{i}^{T} \mathbf{P}_{i}\left(\mathbf{K}_{i} \delta_{i}(\mathbf{x}, t)\right)\right) \\
= & \frac{\partial V(\mathbf{x})}{\partial \mathbf{x}}\left(\mathbf{f}(\mathbf{x})+\sum_{i=1}^{2} \mathbf{g}_{i}(\mathbf{x}) \alpha_{i}(\mathbf{x})\right)+\frac{\partial V(\mathbf{x}) \sum_{i=1}^{2} \mathbf{g}_{i}(\mathbf{x})\left(\mathbf{V}_{i} e r_{i}(t)\right)}{\partial \mathbf{x}} \quad \frac{\partial V(\mathbf{x})}{\partial \mathbf{x}} \mathbf{g}_{i}(\mathbf{x})\left|p_{i}(\mathbf{x})\right| \\
& +m_{i} \sum_{i=1}^{2}\left(-\frac{\partial V(\mathbf{x})}{\partial \mathbf{x}} \mathbf{g}_{i}(\mathbf{x}) p_{i}(\mathbf{x}) \tanh \left(\frac{(\partial V(\mathbf{x}) / \partial x) \mathbf{g}_{i}(\mathbf{x}) p_{i}(\mathbf{x})}{\xi_{i}}\right)\right) \\
& -\sum_{i=1}^{m}\left(e r_{i}^{T} \mathbf{Q}_{i} e r_{i}\right)-2 \sum_{i=1}^{2}\left(e r_{i}^{T} \mathbf{P}_{i}\left(\mathbf{K}_{i} \delta_{i}(\mathbf{x}, t)\right)\right) .
\end{aligned}
$$

According to Lemma 11, the following inequality holds:

$$
0 \leq|x|-x \tanh \left(\frac{x}{\varepsilon}\right) \leq \frac{1}{2} \varepsilon
$$

That is,

$$
\begin{aligned}
& \frac{\partial V(\mathbf{x})}{\partial \mathbf{x}} \mathbf{g}_{i}(\mathbf{x})\left|p_{i}(\mathbf{x})\right| \\
& \quad-\frac{\partial V(\mathbf{x})}{\partial \mathbf{x}} \mathbf{g}_{i}(\mathbf{x}) p_{i}(\mathbf{x}) \tanh \left(\frac{(\partial V(x) / \partial x) \mathbf{g}_{i}(\mathbf{x}) p_{i}(\mathbf{x})}{\xi_{i}}\right) \\
& \quad \leq \frac{1}{2} \xi_{i} .
\end{aligned}
$$

Substituting (46) into (44), we can get

$$
\begin{aligned}
\dot{W} \leq & \frac{\partial V(\mathbf{x})}{\partial \mathbf{x}}\left(\mathbf{f}(\mathbf{x})+\sum_{i=1}^{2} \mathbf{g}_{i}(\mathbf{x}) \alpha_{i}(\mathbf{x})\right) \\
& +\frac{\partial V(\mathbf{x})}{\partial \mathbf{x}} \sum_{i=1}^{2} \mathbf{g}_{i}(\mathbf{x})\left(\mathbf{V}_{i} e r_{i}(t)\right)+m_{i} \frac{1}{2} \xi_{i} \\
& -\sum_{i=1}^{m}\left(e r_{i}^{T} \mathbf{Q}_{i} e r_{i}\right)-2 \sum_{i=1}^{2}\left(e r_{i}^{T} \mathbf{P}_{i}\left(\mathbf{K}_{i} \delta_{i}(\mathbf{x}, t)\right)\right) .
\end{aligned}
$$

With the application of (14) in Assumption 2 into (47), we can obtain

$$
\begin{aligned}
\dot{W} \leq & -d_{0}(\|\mathbf{x}\|)+\frac{\partial V(\mathbf{x})}{\partial \mathbf{x}} \sum_{i=1}^{2} \mathbf{g}_{i}(\mathbf{x})\left(\mathbf{V}_{i} e r_{i}(t)\right) \\
& +m_{i} \frac{1}{2} \xi_{i}-\sum_{i=1}^{m}\left(e r_{i}^{T} \mathbf{Q}_{i} e r_{i}\right) \\
& -2 \sum_{i=1}^{2}\left(e r_{i}^{T} \mathbf{P}_{i}\left(\mathbf{K}_{i} \delta_{i}(\mathbf{x}, t)\right)\right) \\
\leq & -d_{0}(\|\mathbf{x}\|)+\left|\frac{\partial V(\mathbf{x})}{\partial \mathbf{x}} \sum_{i=1}^{2} \mathbf{g}_{i}(\mathbf{x})\right|^{2}\left|\mathbf{V}_{i} e r_{i}(t)\right| \\
& +m_{i} \frac{1}{2} \xi_{i}-\sum_{i=1}^{m}\left(e r_{i}^{T} \mathbf{Q}_{i} e r_{i}\right) \\
& -2 \sum_{i=1}^{2}\left(e r_{i}^{T} \mathbf{P}_{i}\left(\mathbf{K}_{i} \delta_{i}(\mathbf{x}, t)\right)\right) .
\end{aligned}
$$

Assuming that $\lambda_{\min }\left(\mathbf{Q}_{i}\right)$ represent the minimal eigenvalue of the matrix $\mathbf{Q}_{i},(48)$ can be given by

$$
\begin{aligned}
\dot{W} \leq & -d_{0}(\|\mathbf{x}\|)+\left|\frac{\partial V(\mathbf{x})}{\partial \mathbf{x}} \sum_{i=1}^{2} \mathbf{g}_{i}(\mathbf{x})\right|^{2}\left|\mathbf{V}_{i} e r_{i}(t)\right| \\
& +m_{i} \frac{1}{2} \xi_{i}-\sum_{i=1}^{m} \lambda_{\min }\left(\mathbf{Q}_{i}\right)\left\|e r_{i}\right\|^{2} \\
& +\sum_{i=1}^{2}\left(2 \mu_{i}\left\|\mathbf{P}_{i} \mathbf{K}_{i}\right\|^{2}\left\|e r_{i}^{T}\right\|^{2}+\frac{1}{2 \mu_{i}}\left|m_{i} p_{i}(\mathbf{x})\right|^{2}\right),
\end{aligned}
$$

where $\mu_{i}$ is a positive number. 
In terms of permanent establishment inequality $2 a b \leq$ $c a^{2}+c^{-1} b^{2},(49)$ is rewritten as

$$
\begin{aligned}
\dot{W} \leq & -d_{0}(\|\mathbf{x}\|)+\left(\left|\frac{\partial V(\mathbf{x})}{\partial \mathbf{x}} \sum_{i=1}^{2} \mathbf{g}_{i}(\mathbf{x})\right|^{2}+\frac{1}{4} \sum_{i=1}^{2}\left\|\mathbf{V}_{i}\right\|^{2}\left\|e r_{i}\right\|^{2}\right) \\
& +m_{i} \frac{1}{2} \xi_{i}-\sum_{i=1}^{2} \lambda_{\min }\left(\mathbf{Q}_{i}\right)\left\|e r_{i}\right\|^{2} \\
& +\sum_{i=1}^{2}\left(2 \mu_{i}\left\|\mathbf{P}_{i} \mathbf{K}_{i}\right\|^{2}\left\|e r_{i}^{T}\right\|^{2}+\frac{1}{2 \mu_{i}}\left|m_{i} p_{i}(\mathbf{x})\right|^{2}\right) .
\end{aligned}
$$

With the application of (15) in Assumption 2 into (50), we can obtain

$$
\begin{aligned}
\dot{W} \leq & -d_{0}(\|\mathbf{x}\|)+\left(d_{0}(\|\mathbf{x}\|)+\frac{1}{4} \sum_{i=1}^{2}\left\|\mathbf{V}_{i}\right\|^{2}\left\|e r_{i}\right\|^{2}\right) \\
& +m_{i} \frac{1}{2} \xi_{i}-\sum_{i=1}^{2} \lambda_{\min }\left(\mathbf{Q}_{i}\right)\left\|e r_{i}\right\|^{2} \\
& +\sum_{i=1}^{2}\left(2 \mu_{i}\left\|\mathbf{P}_{i} \mathbf{K}_{i}\right\|^{2}\left\|e r_{i}^{T}\right\|^{2}+\frac{1}{2 \mu_{i}}\left|m_{i} p_{i}(\mathbf{x})\right|^{2}\right) \\
= & \frac{1}{4} \sum_{i=1}^{2}\left\|\mathbf{V}_{i}\right\|^{2}\left\|e r_{i}\right\|^{2}+m_{i} \frac{1}{2} \xi_{i}-\sum_{i=1}^{2} \lambda_{\min }\left(\mathbf{Q}_{i}\right)\left\|e r_{i}\right\|^{2} \\
& +\sum_{i=1}^{2}\left(2 \mu_{i}\left\|\mathbf{P}_{i} \mathbf{K}_{i}\right\|^{2}\left\|e r_{i}^{T}\right\|^{2}+\frac{1}{2 \mu_{i}}\left|m_{i} p_{i}(\mathbf{x})\right|^{2}\right) \\
\leq & \frac{1}{4} \sum_{i=1}^{2}\left\|\mathbf{V}_{i}\right\|^{2}\left\|e r_{i}\right\|^{2}-\sum_{i=1}^{2} \lambda_{\min }\left(\mathbf{Q}_{i}\right)\left\|e r_{i}\right\|^{2} \\
& +m_{i} \frac{1}{2} \xi_{i}+\sum_{i=1}^{2}\left(2 \mu_{i}\left\|\mathbf{P}_{i} \mathbf{K}_{i}\right\|^{2}\left\|e r_{i}^{T}\right\|^{2}+\frac{m_{i}}{2 \mu_{i}}\left|p_{\max }\right|^{2}\right),
\end{aligned}
$$

where $\left|p_{\max }\right|$ indicates the maximum value of $\left|p_{i}(x)\right|$.

Suppose that $d_{i}=\lambda_{\text {min }}\left(\mathbf{Q}_{i}\right)-(1 / 4)\left\|V_{i}\right\|^{2}-2 \mu_{i}\left\|\mathbf{P}_{i} \mathbf{K}_{i}\right\|^{2}>0$ and choose proper $\xi_{i}$ to satisfy

$$
\dot{W} \leq-\sum_{i=1}^{m} d_{i}\left\|\mathbf{e}_{\mathrm{i}}\right\|^{2}+m_{i} \frac{1}{2} \xi_{i}+\sum_{i=1}^{2}\left(\frac{m_{i}}{2 \mu_{i}}\left|p_{\max }\right|^{2}\right) \leq 0 .
$$

As can be seen above, the proposed controller can ensure all the signals of the closed-loop system uniformly bounded. Consequently, the paper comes to the following conclusion.

Theorem 13. There exist positive definite matrices $\mathbf{P}_{i}$ and $\mathbf{Q}_{i}$ satisfying formula (21) and nonzero constant vector $\mathbf{K}_{i} \in$ $\mathbf{R}^{q}$ satisfying Assumption 3, such that the matrix $\mathbf{W}_{i}-\mathbf{K}_{i} \mathbf{V}_{i}$ is Hurwitz. Furthermore, the formula (52) holds. For the multivariable nonlinear system (9) and exosystem (10) satisfying the Assumptions (1) to (5), the nonlinear multivariable internal models (18) and (22) and the input feedback control (25) can make the closed-loop system globally uniformly bounded.

\section{Numerical Simulations and Analysis}

To illustrate the performance of the present control algorithm, some numerical simulations are performed in the section. The whole simulation time is 5 seconds with the sampling interval $0.001 \mathrm{~s}$. The simulation parameters are chosen as follows.

For the grid, the rms value of network voltage $e_{0}=380 \mathrm{~V}$ the synchronous angular velocity $\omega=100 \pi \mathrm{rad} / \mathrm{s}$. For the three-phase grid-connected inverters, the filter inductance $L=1.0 \mathrm{mH}$ and equivalent series resistance $R=0.02 \Omega$.

Substituting the relevant parameters into the original state equation (9), the nonlinear system (9) can be described by

$$
\dot{\mathbf{x}}=\mathbf{f}(\mathbf{x})+\sum_{i=1}^{2} \mathbf{g}_{i}(\mathbf{x})\left(\mathbf{v}_{i}-\mathbf{e}_{i 0}-D_{i}(\mathbf{w})+\delta_{i}(\mathbf{x}, t)\right),
$$

$$
1 \leq i \leq 2 \text {, }
$$

where $\mathbf{x}=\left[x_{1}, x_{2}\right]^{T}, \mathbf{f}(\mathbf{x})=\left[\begin{array}{l}-20 x_{1}+100 \pi x_{2} \\ -100 \pi x_{1}-20 x_{2}\end{array}\right], \mathbf{g}_{1}(\mathbf{x})=$ $\mathbf{g}_{2}(\mathbf{x})=1000$, and $\left[e_{10}, e_{20}\right]=[380,0]$. The control input $\mathbf{v}=$ $\left[v_{1}, v_{2}\right]^{T}$. It is thus clear that the system (53) has two-variable input, and the conventional single-input algorithm cannot solve the problem.

Example 14. The network is immersed 3\% 3rd harmonics to fundamental wave.

The exosystem matrices for exogenous disturbance $\mathbf{D}(\mathbf{w})$ represented in (10) and (18) are given as $\mathbf{W}_{1}=\mathbf{W}_{2}=$ $\left[\begin{array}{cc}0 & 2 \omega \\ -2 \omega & 0\end{array}\right], \mathbf{V}_{1}=\mathbf{V}_{2}=\left[\begin{array}{cc}11.4 & 0\end{array}\right]$, and the uncertain items satisfy

$$
\delta_{1}(\mathbf{x}, t)=x_{1} \sin \left(x_{1} t\right), \quad \delta_{2}(\mathbf{x}, t)=x_{2} \sin \left(x_{2} t\right) .
$$

It is worth noticing that the rotating electrical angular velocity of the 3rd harmonics in stationary $a b c$ reference frame is in accordance with that of the 2nd harmonics in rotating $d q$ reference frame of fundamental wave. Hence, $\mathbf{W}_{1}=\mathbf{W}_{2}=\left[\begin{array}{cc}0 & 2 \omega \\ -2 \omega & 0\end{array}\right]$ in $d q$ coordinated system represents the $3 \mathrm{rd}$ harmonics in stationary $a b c$ coordinated system. Furthermore, the values of $\mathbf{V}_{1}$ and $\mathbf{V}_{2}$ represent that the network is immersed 3\% 3rd harmonics to fundamental wave.

In terms of (33) and (38), the control law of the nominal system is given by

$$
\begin{aligned}
\alpha_{1}(\mathbf{x}) & =-L z_{1}+R x_{1}-L \omega x_{2}+L \dot{x}_{1 \mathrm{ref}} \\
& =-0.001\left(x_{1}-x_{1 \mathrm{ref}}\right)+0.02 x_{1}-0.1 \pi x_{2}+0.001 \dot{x}_{1 \mathrm{ref}}, \\
\alpha_{2}(\mathbf{x}) & =-L e z_{2}+R x_{2}+L \omega x_{1}+L \dot{x}_{2 \mathrm{ref}} \\
& =-0.001\left(x_{2}-x_{2 \mathrm{ref}}\right)+0.02 x_{1}+0.1 \pi x_{2}+0.001 \dot{x}_{2 \mathrm{ref}} .
\end{aligned}
$$

Supposing that

$$
V(\mathbf{x})=\frac{1}{2} c_{1}\left(x_{1}-x_{1 \mathrm{ref}}\right)^{2}+\frac{1}{2} c_{2}\left(x_{2}-x_{2 \mathrm{ref}}\right)^{2},
$$


we can obtain

$$
\begin{aligned}
& \frac{\partial V(\mathbf{x})}{\partial \mathbf{x}}\left(\mathbf{f}(\mathbf{x})+\sum_{i=1}^{2} \mathbf{g}_{i}(\mathbf{x}) \boldsymbol{\alpha}_{i}\right) \\
& =-\left(x_{1}-x_{1 \mathrm{ref}}\right)^{2}-\left(x_{2}-x_{2 \mathrm{ref}}\right)^{2}, \\
& \frac{\partial \mathbf{V}(\mathbf{x})}{\partial \mathbf{x}} \sum_{i=1}^{2} \mathbf{g}_{i}(\mathbf{x}) \\
& =\frac{c_{1}\left(x_{1}-x_{1 \mathrm{ref}}\right)}{L}+\frac{c_{2}\left(x_{2}-x_{2 \mathrm{ref}}\right)}{L} .
\end{aligned}
$$

Supposing $\mathbf{x}^{\prime}=\left[\begin{array}{ll}\left(x_{1}-x_{1 \mathrm{ref}}\right) & \left(x_{2}-x_{2 \mathrm{ref}}\right)^{T}\end{array}\right]$, in terms of (56), (57) and (58), and choosing $c_{1}=L$ and $c_{2}=L$ make the following inequalities hold

$$
\begin{gathered}
\frac{1}{2} L\left\|\mathbf{x}^{\prime}\right\|^{2} \leq V(\mathbf{x}) \leq L\left\|\mathbf{x}^{\prime}\right\|^{2}, \\
\frac{\partial V(\mathbf{x})}{\partial \mathbf{x}}\left(\mathbf{f}(\mathbf{x})+\sum_{i=1}^{2} \mathbf{g}_{i}(\mathbf{x}) \alpha_{i}\right) \leq-\left\|\mathbf{x}^{\prime}\right\|^{2}, \\
\left|\frac{\partial \mathbf{V}(\mathbf{x})}{\partial \mathbf{x}} \sum_{i=1}^{m} \mathbf{g}_{i}(\mathbf{x})\right|^{2} \leq\left\|\mathbf{x}^{\prime}\right\|^{2} .
\end{gathered}
$$

That is, Assumption 2 is satisfied.

Choosing

$$
r_{1}(\mathbf{x})=9 L x_{1}, \quad r_{2}(\mathbf{x})=9 L x_{1},
$$

we can obtain

$$
\begin{aligned}
& \mathbf{K}_{1}=\frac{\partial r_{1}(\mathbf{x})}{\partial \mathbf{x}} \mathbf{g}_{1}(\mathbf{x})=\left[\begin{array}{ll}
9 & 0
\end{array}\right]^{T}, \\
& \mathbf{K}_{2}=\frac{\partial r_{2}(\mathbf{x})}{\partial \mathbf{x}} \mathbf{g}_{2}(\mathbf{x})=\left[\begin{array}{ll}
9 & 0
\end{array}\right]^{T} .
\end{aligned}
$$

Therefore, Assumption 3 holds.

According to (61), we can obtain

$$
\begin{aligned}
& \mathbf{W}_{1}-\mathbf{K}_{1} \mathbf{V}_{1}=\left[\begin{array}{lc}
-102.6000 & 628.3185 \\
-628.3185 & 0
\end{array}\right], \\
& \mathbf{W}_{2}-\mathbf{K}_{2} \mathbf{V}_{2}=\left[\begin{array}{cc}
-102.6000 & 628.3185 \\
-628.3185 & 0
\end{array}\right] .
\end{aligned}
$$

Furthermore, let $\mathbf{P}_{1}=\mathbf{P}_{2}=\left[\begin{array}{cc}4 & -2 \\ 2 & 4\end{array}\right]$; substituting (62) and (63) into (21), respectively, we obtain

$$
\begin{aligned}
\mathbf{Q}_{1} & =\left[\begin{array}{cc}
820.8000 & -205.2000 \\
205.2000 & 0
\end{array}\right], \\
\mathbf{Q}_{2} & =\left[\begin{array}{cc}
820.8000 & -205.2000 \\
205.2000 & 0
\end{array}\right] .
\end{aligned}
$$

Let $\mu_{1}=\mu_{2}=0.005$; substituting $\mathbf{Q}_{i}, \mathbf{V}_{i}, \mathbf{P}_{i}, \mathbf{K}_{i}$, and $\mu_{i}$ into (52), through some arithmetical operations, we can obtain

$$
\begin{aligned}
d_{1} & =\lambda_{\text {min }}\left(\mathbf{Q}_{1}\right)-\frac{1}{4}\left\|\mathbf{V}_{1}\right\|^{2}-2 \mu_{1}\left\|\mathbf{P}_{1} \mathbf{K}_{1}\right\|^{2} \\
& =54.9832-\frac{1}{4} \times 129.9600-2 \mu_{1} \times 1620>0, \\
d_{2} & =\lambda_{\min }\left(\mathbf{Q}_{2}\right)-\frac{1}{4}\left\|\mathbf{V}_{2}\right\|^{2}-2 \mu_{2}\left\|\mathbf{P}_{2} \mathbf{K}_{2}\right\|^{2} \\
& =54.9832-\frac{1}{4} \times 129.9600-2 \mu_{2} \times 1620>0 .
\end{aligned}
$$

Hence, through the presented algorithm, the final internal models of disturbance and uncertain items are given by

$$
\begin{aligned}
& \widehat{D}_{1}(\mathbf{w})=\mathbf{V}_{1} \widehat{\mathbf{w}}_{1}(t), \quad \widehat{D}_{2}(\mathbf{w})=\mathbf{V}_{2} \widehat{\mathbf{w}}_{2}(t), \\
& \widehat{\mathbf{w}}_{1}(t)=\widehat{\boldsymbol{\eta}}_{1}(t)+\mathbf{r}_{1}(x), \quad \widehat{\mathbf{w}}_{2}(t)=\widehat{\boldsymbol{\eta}}_{2}(t)+\mathbf{r}_{2}(\mathbf{x}), \\
& \dot{\hat{\eta}}_{11}(t)=-102.6 \hat{\eta}_{11}(t)-0.7434 x_{1} \\
& +628.3185 \hat{\eta}_{12}(t)-9 u_{1}-2.8274 x_{2}, \\
& \dot{\hat{\eta}}_{12}(t)=-628.3185 \widehat{\eta}_{11}-5.6549 x_{1}, \\
& \dot{\hat{\eta}}_{21}(t)=-102.6 \widehat{\eta}_{21}(t)+628.3185 \widehat{\eta}_{22}(t)-9 u_{1} \\
& +1.9036 x_{1}+0.18 x_{2}, \\
& \dot{\hat{\eta}}_{22}(t)=-628.3185 \widehat{\eta}_{21}-5.6549 x_{1} \text {, } \\
& \widehat{\delta}_{1}(\mathbf{x}, t)=m_{1} \mathbf{K}_{1} p_{1}(\mathbf{x}) \tanh \left(\frac{\left[\begin{array}{ll}
\widehat{\eta}_{11} & \widehat{\eta}_{12}
\end{array}\right] \mathbf{P}_{1}^{T} \mathbf{K}_{1} p_{1}(\mathbf{x})}{\lambda_{1}}\right) \\
& =\left[\begin{array}{c}
9 \tanh \left(\frac{\left(36 \hat{\eta}_{11}-18 \widehat{\eta}_{12}\right) x_{1}}{\lambda_{1}}\right) \\
0
\end{array}\right] \text {, } \\
& \widehat{\delta}_{2}(\mathbf{x}, t)=m_{2} \mathbf{K}_{2} p_{2}(\mathbf{x}) \tanh \left(\frac{\left[\begin{array}{ll}
\widehat{\eta}_{3} & \widehat{\eta}_{4}
\end{array}\right] \mathbf{P}_{2}^{T} \mathbf{K}_{2} p_{2}(\mathbf{x})}{\lambda_{2}}\right) \\
& =\left[\begin{array}{c}
9 \tanh \left(\frac{\left(36 \hat{\eta}_{21}-18 \widehat{\eta}_{22}\right) x_{2}}{\lambda_{2}}\right) \\
0
\end{array}\right] \text {. }
\end{aligned}
$$

Choosing $m_{1}=m_{2}=1, \xi_{1}=\xi_{2}=0.1$, the controller is designed as

$$
\begin{aligned}
\alpha_{1}(\mathbf{x}) & =-\frac{L e z_{1}}{c_{1}}+R x_{1}-L \omega x_{2}+L \dot{x}_{1 \mathrm{ref}} \\
& =-\left(x_{1}-x_{1 \mathrm{ref}}\right)+0.02 x_{1}-0.3142 x_{2}, \\
\alpha_{2}(\mathbf{x}) & =-\frac{L e z_{2}}{c_{2}}+R x_{2}+L \omega x_{1}+L \dot{x}_{2 \mathrm{ref}} \\
& =-\left(x_{2}-x_{2 \mathrm{ref}}\right)+0.02 x_{2}+0.3142 x_{1},
\end{aligned}
$$




$$
\begin{aligned}
v_{1}^{\prime}= & \alpha_{1}(\mathbf{x})-\widehat{D}_{1}(\mathbf{w}) \\
& +m_{1} p_{1}(\mathbf{x}) \tanh \left(\frac{(\partial V(\mathbf{x}) / \partial x) g_{1}(\mathbf{x}) p_{1}(\mathbf{x})}{\xi_{1}}\right) \\
= & -\left(x_{1}-x_{1 \mathrm{ref}}\right)+0.02 x_{1}-0.3142 x_{2} \\
& -11.4\left(\widehat{\eta}_{11}(t)+0.009 x_{1}\right) \\
& +x_{1} \tanh \left(\frac{\left(x_{1}-x_{1 \mathrm{ref}}\right) x_{1}}{\xi_{1}}\right)-380, \\
v_{2}^{\prime}= & \alpha_{2}(\mathbf{x})-\widehat{D}_{2}(\mathbf{w}) \\
& +m_{2} p_{2}(\mathbf{x}) \tanh \left(\frac{(\partial V(\mathbf{x}) / \partial x) g_{2}(\mathbf{x}) p_{2}(\mathbf{x})}{\xi_{2}}\right) \\
= & -\left(x_{2}-x_{2 \mathrm{ref}}\right)+0.02 x_{2}+0.3142 x_{1} \\
& -11.4\left(\widehat{\eta}_{21}(t)+0.009 x_{1}\right) \\
& +x_{2} \tanh \left(\frac{\left(x_{2}-x_{2 \mathrm{ref}}\right) x_{2}}{\xi_{2}}\right) .
\end{aligned}
$$

Let the initial condition of the simulation be $x(0)=$ $\left[\begin{array}{ll}0 & 0\end{array}\right]^{T}, \widehat{\eta}(0)=\left[\begin{array}{llll}0 & 0 & 0 & 0\end{array}\right]^{T}, \widehat{w}(0)=\left[\begin{array}{ll}1 & 0\end{array}\right]^{T}$. The reference values of the output current are chosen as $x_{\text {ref }}=$ $[4,0]^{T}$. Figures 3, 4, 5, and 6 show the simulation results. Figure 3 demonstrates the system states (output currents in $d q$ reference frame). Figure 4 indicates the control inputs in $d q$ axis, respectively. The disturbances produced by the exosystem and their estimates are shown in Figure 5. Figure 6 demonstrates the estimating errors under the existence of exosystem disturbances and uncertain items. As shown in Figure 3, the system states are asymptotically convergence to the expected references under the existence of exogenous disturbances and uncertain modeling perturbations. Figures 5 and 6 also indicate that the designed internal models can produce the exogenous disturbance successfully and the estimating errors converge to zero.

Example 15. The network is immersed 1.5\% 3rd harmonics and $1 \% 5$ th harmonics to fundamental wave.

The exosystem matrices for exogenous disturbance $\mathbf{D}(w)$ represented in (10) and (18) are given as $\mathbf{W}=$ $\left[\begin{array}{cccc}0 & 2 \omega & 0 & 0 \\ -2 \omega & 0 & 0 & 0 \\ 0 & 0 & 0 & 4 \omega \\ 0 & 0 & -4 \omega & 0\end{array}\right], \mathbf{V}_{1}=\mathbf{V}_{2}=\left[\begin{array}{llll}5.7 & 0 & 3.8 & 0\end{array}\right]$, and the uncertain items also satisfy

$$
\delta_{1}(\mathbf{x}, t)=x_{1} \sin \left(x_{1} t\right), \quad \delta_{2}(\mathbf{x}, t)=x_{2} \sin \left(x_{2} t\right) .
$$

The design of the nominal system and the verification of Assumption 2 are the same as in Example 14, their derivation processes are no longer described in detail.

Choosing

$$
r_{1}(\mathbf{x})=r_{2}(\mathbf{x})=\left[\begin{array}{ll}
9 L x_{1} & 9 L x_{1}
\end{array}\right]^{T},
$$

we can obtain:

$$
K_{1}=K_{2}=\frac{\partial r_{1}(\mathbf{x})}{\partial x} \mathbf{g}_{1}(\mathbf{x})=\left[\begin{array}{llll}
9 & 0 & 9 & 0
\end{array}\right]^{T}
$$

Therefore, Assumption 3 holds.

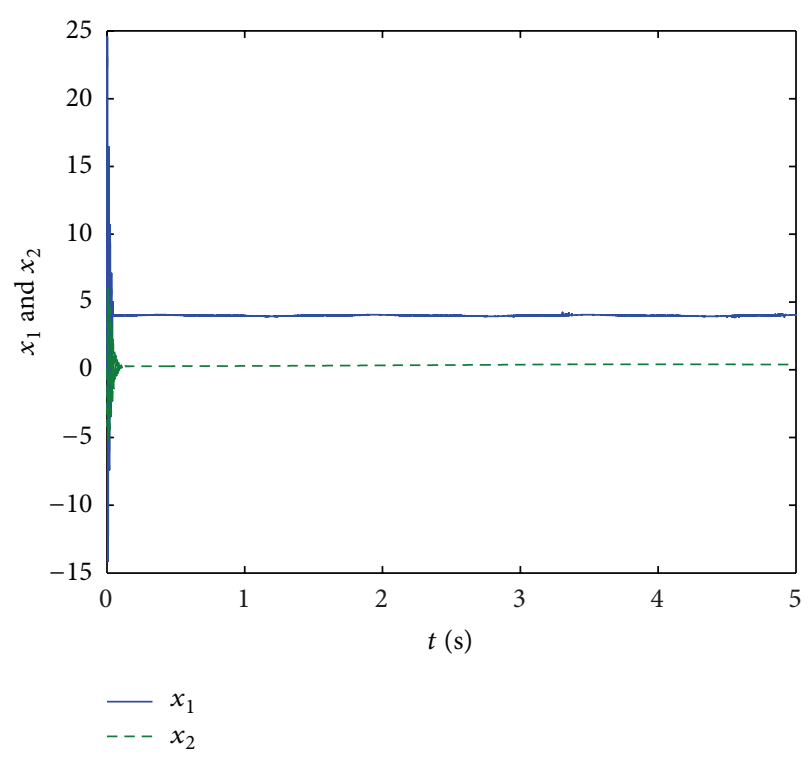

Figure 3: System states (output currents in $d q$ reference frame).

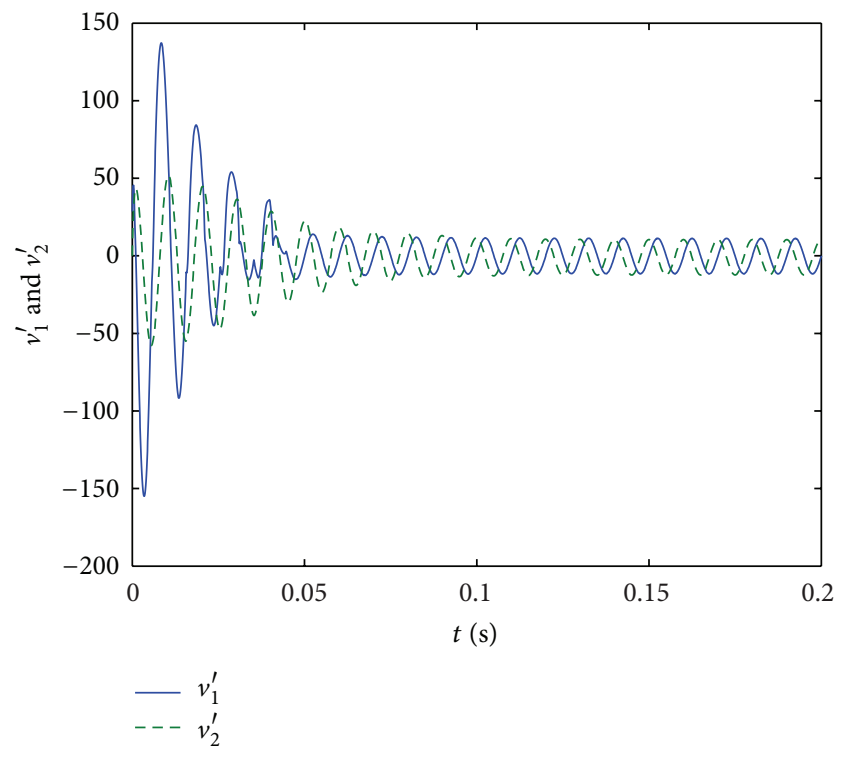

FIGURE 4: Control inputs in $d q$ axis, respectively.

In terms of (71), we can obtain

$$
\begin{aligned}
\mathbf{W}_{1} & -\mathbf{K}_{1} \mathbf{V}_{1} \\
& =\mathbf{W}_{2}-\mathbf{K}_{2} \mathbf{V}_{2} \\
& =\left[\begin{array}{cccc}
-51.3000 & 628.3185 & -34.2000 & 0 \\
-628.3185 & 0 & 0 & 0 \\
-51.3000 & 0 & -34.2000 & 1256.637 \\
0 & 0 & -1256.637 & 0
\end{array}\right] .
\end{aligned}
$$




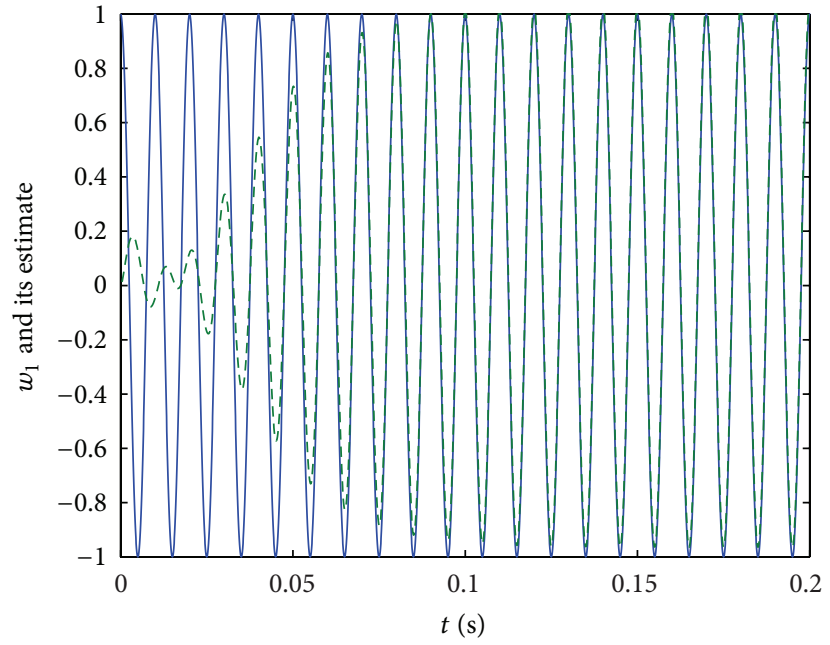

$-w_{1}$

- - Estimate

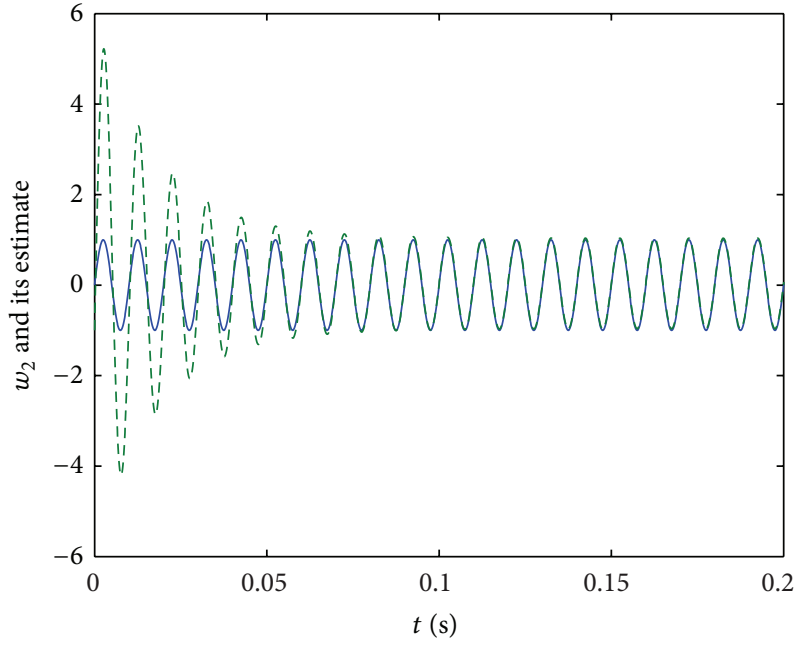

$-w_{2}$

- - Estimate

(a)

(b)

FIGURE 5: Exogenous disturbances of $w_{1}$ and $w_{2}$ and their estimates.

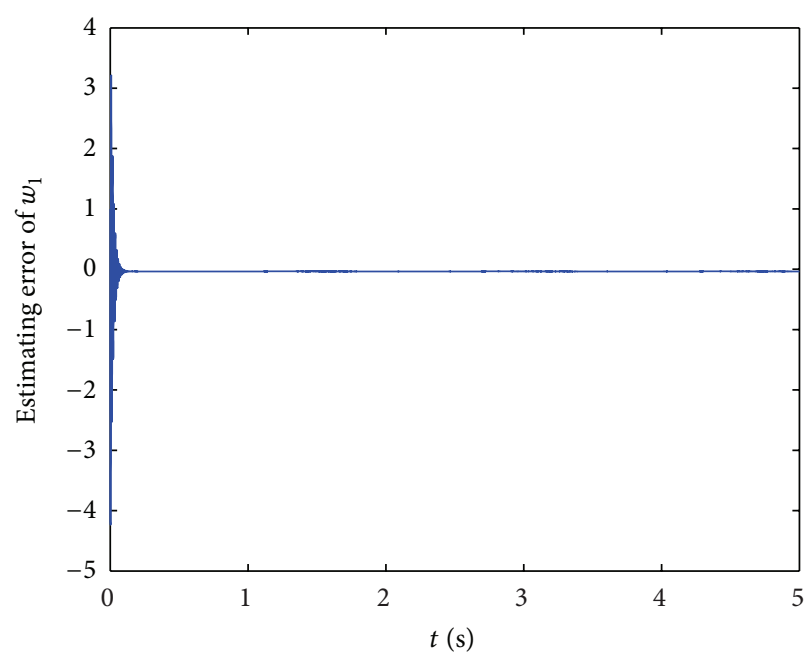

(a)

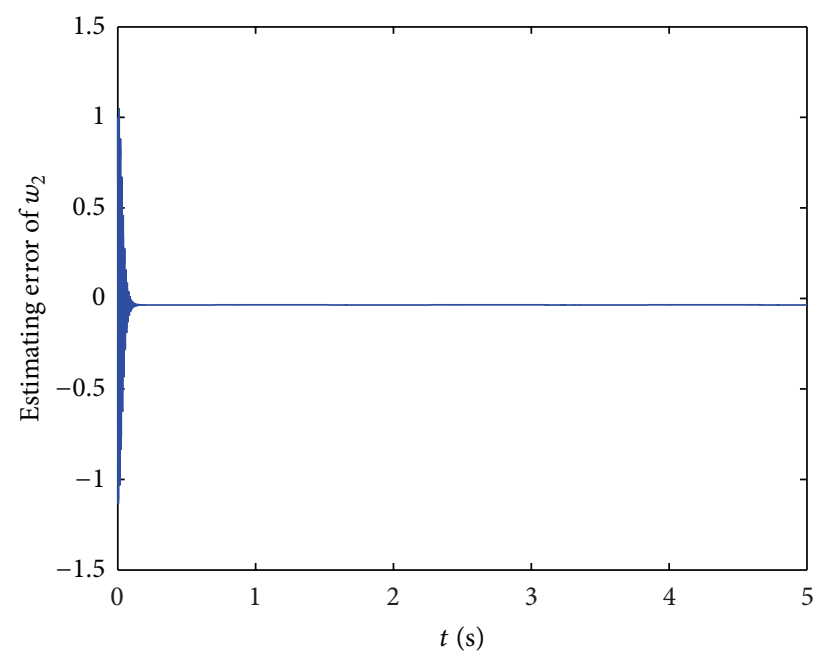

(b)

FIGURE 6: Estimating errors of $w_{1}$ and $w_{2}$.

Let

$$
\begin{aligned}
\mathbf{P}_{1} & =\mathbf{P}_{2} \\
& =\left[\begin{array}{cccc}
3.970777 & -0.159154 & -0.072142 & -0.300626 \\
-0.159154 & 4.137151 & 0.070735 & 0.045912 \\
-0.072142 & 0.070735 & 5.920095 & -0.079577 \\
-0.300626 & 0.045912 & -0.07957 & 6.010020
\end{array}\right] ;
\end{aligned}
$$

substituting (73) into (21), we obtain

$$
\begin{aligned}
\mathbf{Q}_{1} & =\mathbf{Q}_{2} \\
& =\left[\begin{array}{cccc}
200.0011 & 100.0000 & 99.9996 & 99.9993 \\
100.0000 & 199.9988 & 99.9989 & 100.0007 \\
99.9996 & 99.9989 & 200.0100 & 100.0001 \\
99.9996 & 100.0007 & 100.0004 & 199.9900
\end{array}\right] .
\end{aligned}
$$




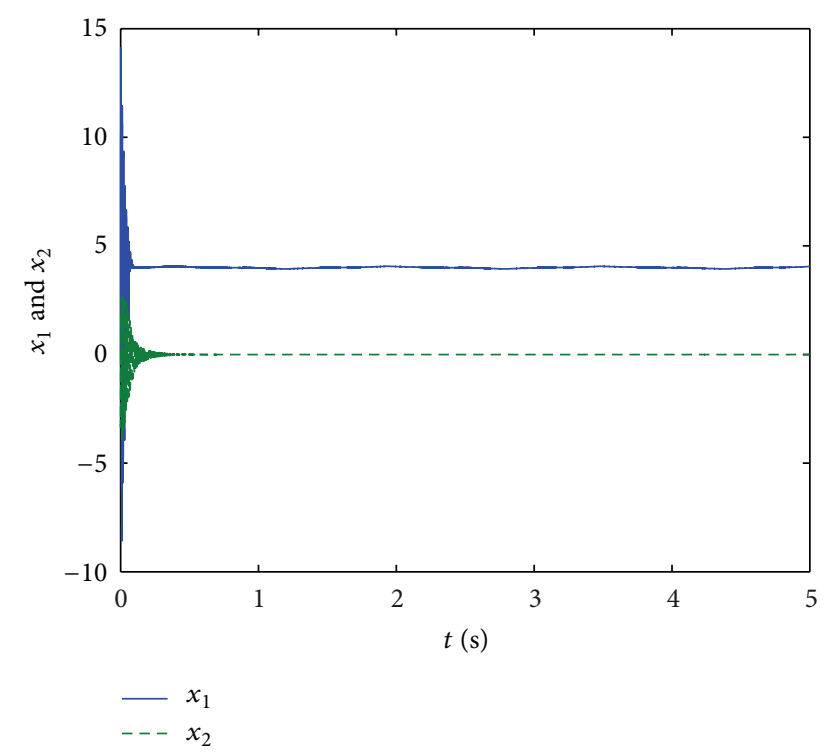

FIGURE 7: System states (output currents in $d q$ reference frame).

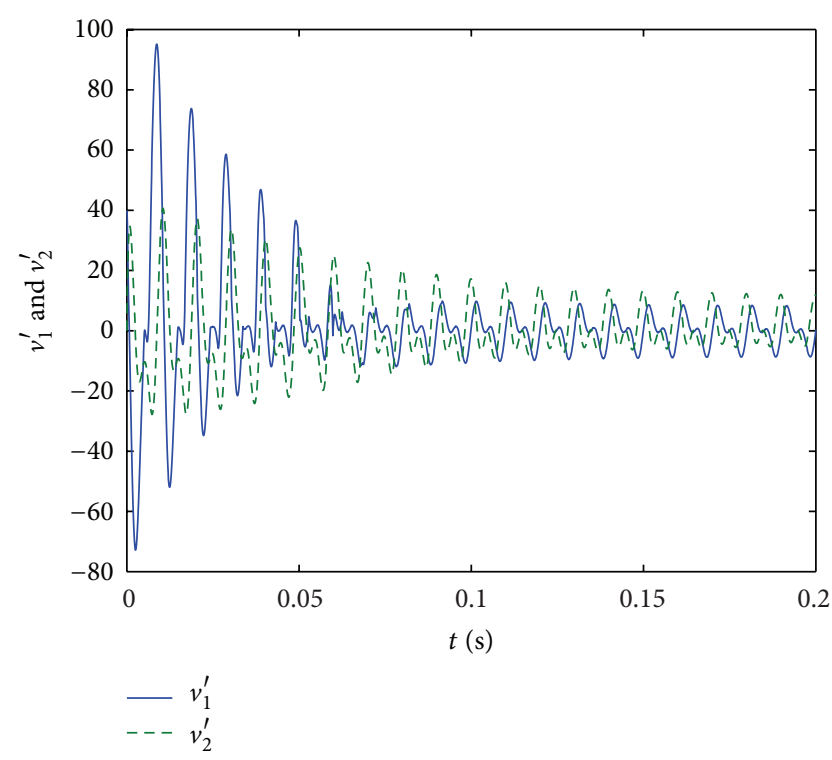

FIgURE 8: Control inputs in $d q$ axis, respectively.

Let $\mu_{1}=\mu_{2}=0.01$; substituting $\mathbf{Q}_{i}, \mathbf{V}_{i}, \mathbf{P}_{i}, \mathbf{K}_{i}$, and $\mu_{i}$ into (52), through some arithmetical operations, we can obtain

$$
\begin{aligned}
d_{1} & =\lambda_{\min }\left(\mathbf{Q}_{1}\right)-\frac{1}{4}\left\|\mathbf{V}_{1}\right\|^{2}-2 \mu_{1}\left\|P_{1} K_{1}\right\|^{2} \\
& =99.9924-\frac{1}{4} \times 46.93-2 \mu_{1} \times 4013.57>0, \\
d_{2} & =\lambda_{\min }\left(\mathbf{Q}_{2}\right)-\frac{1}{4}\left\|\mathbf{V}_{2}\right\|^{2}-2 \mu_{2}\left\|\mathbf{P}_{2} \mathbf{K}_{2}\right\|^{2} \\
& =99.9924-\frac{1}{4} \times 46.93-2 \mu_{1} \times 4013.57>0 .
\end{aligned}
$$

Hence, through the presented algorithm, the final internal models of disturbance and uncertain items are given by

$$
\begin{aligned}
& \widehat{D}_{1}(\mathbf{w})=\mathbf{V}_{1} \widehat{\mathbf{w}}_{1}(t), \quad \widehat{D}_{2}(\mathbf{w})=\mathbf{V}_{2} \widehat{\mathbf{w}}_{2}(t), \\
& \widehat{\mathbf{w}}_{1}(t)=\widehat{\boldsymbol{\eta}}_{1}(t)+\mathbf{r}_{1}(x), \quad \widehat{\mathbf{w}}_{2}(t)=\widehat{\boldsymbol{\eta}}_{2}(t)+\mathbf{r}_{2}(\mathbf{x}), \\
& \dot{\hat{\eta}}_{11}(t)=-51.3 \widehat{\eta}_{11}+628.3185 \widehat{\eta}_{12}-34.2 \widehat{\eta}_{13} \\
& -9 u_{1}+5.3731 x_{1}-2.8274 x_{2} \text {, } \\
& \dot{\hat{\eta}}_{12}(t)=-628.3185 \widehat{\eta}_{11}-5.6549 x_{1} \text {, } \\
& \dot{\hat{\eta}}_{13}(t)=-51.3 \widehat{\eta}_{11}-34.2 \widehat{\eta}_{13}+1256.637 \widehat{\eta}_{14} \\
& -9 u_{1}-0.2817 x_{1}-2.8274 x_{2} \text {, } \\
& \dot{\hat{\eta}}_{14}(t)=-1256.637 \widehat{\eta}_{13}, \\
& \dot{\hat{\eta}}_{21}(t)=-51.3 \widehat{\eta}_{21}+628.3185 \widehat{\eta}_{22}-34.2 \widehat{\eta}_{23} \\
& -9 u_{2}+2.3657 x_{1}+0.18 x_{2} \text {, } \\
& \dot{\hat{\eta}}_{22}(t)=-628.3185 \widehat{\eta}_{21}-5.6549 x_{1} \text {, } \\
& \dot{\hat{\eta}}_{23}(t)=-51.3 \widehat{\eta}_{21}-34.2 \widehat{\eta}_{23}+1256.637 \widehat{\eta}_{24} \\
& -9 u_{2}+2.3657 x_{1}+0.18 x_{2} \text {, } \\
& \dot{\hat{\eta}}_{24}(t)=-1256.637 \widehat{\eta}_{23}, \\
& \widehat{\delta}_{1}(\mathbf{x}, t)=m_{1} \mathbf{K}_{1} p_{1}(\mathbf{x}) \tanh
\end{aligned}
$$

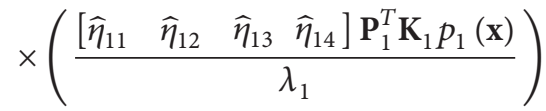$$
=\left[\begin{array}{c}
9 \tanh \left(\frac{\left(\begin{array}{c}
35.0877 \hat{\eta}_{11}-0.7958 \widehat{\eta}_{12} \\
+52.6316 \hat{\eta}_{13}-3.4218 \widehat{\eta}_{14}
\end{array}\right) x_{1}}{\lambda_{1}}\right) \\
0 \\
9 \tanh \left(\frac{\left(\begin{array}{c}
35.0877 \widehat{\eta}_{11}-0.7958 \widehat{\eta}_{12} \\
+52.6316 \hat{\eta}_{13}-3.4218 \widehat{\eta}_{14}
\end{array}\right) x_{1}}{\lambda_{1}}\right) \\
0
\end{array}\right],
$$

$\widehat{\delta}_{2}(\mathbf{x}, t)=m_{2} \mathbf{K}_{2} p_{2}(\mathbf{x}) \tanh$

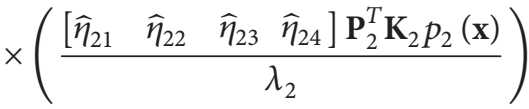

$$
\begin{aligned}
& =\left[\begin{array}{c}
9 \tanh \left(\frac{\left(\begin{array}{c}
35.0877 \widehat{\eta}_{21}-0.7958 \widehat{\eta}_{22} \\
+52.6316 \hat{\eta}_{23}-3.4218 \widehat{\eta}_{24}
\end{array}\right) x_{2}}{\lambda_{2}}\right) \\
0 \\
9 \tanh \left(\frac{\left(\begin{array}{c}
35.0877 \widehat{\eta}_{21}-0.7958 \widehat{\eta}_{22} \\
+52.6316 \hat{\eta}_{23}-3.4218 \widehat{\eta}_{24}
\end{array}\right) x_{2}}{\lambda_{2}}\right) \\
0
\end{array}\right] .
\end{aligned}
$$




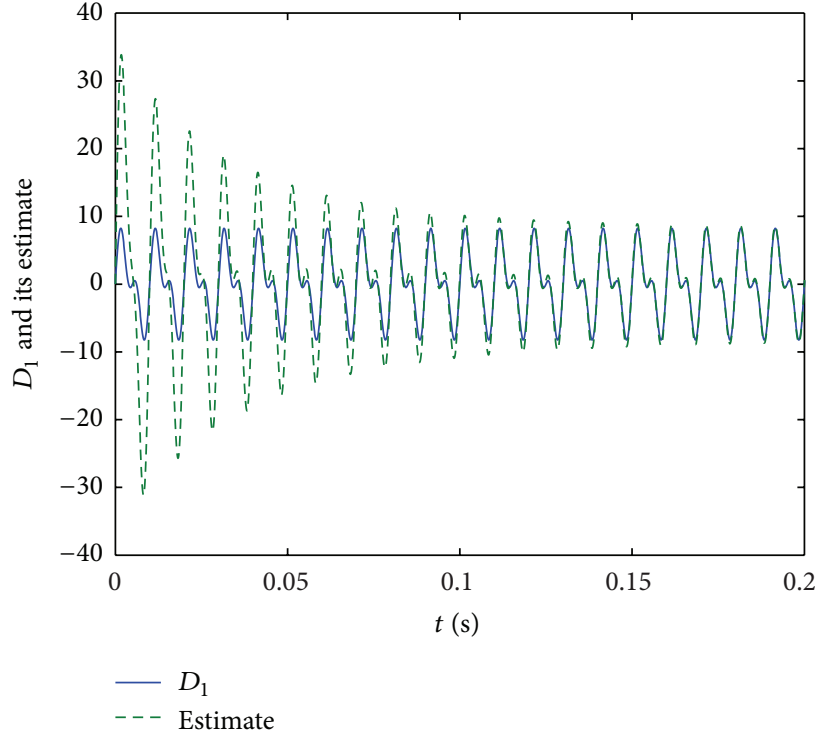

(a)

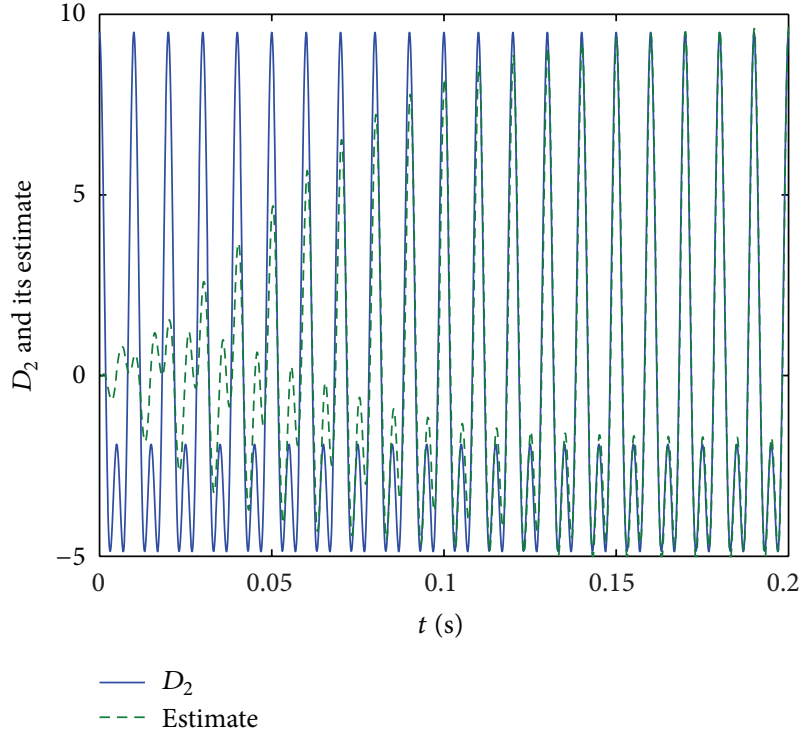

(b)

FIGURE 9: Exogenous disturbances of $w_{1}$ and $w_{2}$ and their estimates.

Choosing $m_{1}=m_{2}=1, \xi_{1}=\xi_{2}=0.1$, the controller is designed as

$$
\begin{aligned}
v_{1}^{\prime}= & \alpha_{1}(\mathbf{x})-\widehat{D}_{1}(\mathbf{w}) \\
& +m_{1} p_{1}(\mathbf{x}) \tanh \left(\frac{(\partial V(\mathbf{x}) / \partial x) \mathbf{g}_{1}(\mathbf{x}) p_{1}(\mathbf{x})}{\xi_{1}}\right) \\
= & -\left(x_{1}-x_{1 \mathrm{ref}}\right)+0.02 x_{1}-0.31415 x_{2} \\
& -5.7\left(\widehat{\eta}_{11}(t)+0.009 x_{1}\right)-3.8 \widehat{\eta}_{13}(t) \\
& +x_{1} \tanh \left(\frac{\left(x_{1}-x_{1 \mathrm{ref}}\right) x_{1}}{\xi_{1}}\right)-380, \\
v_{2}^{\prime}= & \alpha_{2}(\mathbf{x})-\widehat{D}_{2}(\mathbf{w}) \\
& +m_{2} p_{2}(\mathbf{x}) \tanh \left(\frac{(\partial V(x) / \partial x) \mathbf{g}_{2}(\mathbf{x}) p_{2}(\mathbf{x})}{\xi_{2}}\right) \\
= & -\left(x_{2}-x_{2 \mathrm{ref}}\right)+0.02 x_{2}+0.31415 x_{1} \\
& -5.7\left(\widehat{\eta}_{21}(t)+0.009 x_{1}\right)-3.8 \widehat{\eta}_{23}(t) \\
& +x_{2} \tanh \left(\frac{\left(x_{2}-x_{2 \mathrm{ref}}\right) x_{2}}{\xi_{2}}\right) .
\end{aligned}
$$

Let the initial condition of the simulation be $x(0)=$ $\left[\begin{array}{ll}0 & 0\end{array}\right]^{T}, \widehat{\eta}(0)=\left[\begin{array}{cccccccc}0 & 0 & 0 & 0 & 0 & 0 & 0 & 0\end{array}\right]^{T}$, and $\widehat{w}(0)=$ $\left[\begin{array}{llll}1 & 0 & 1 & 0\end{array}\right]^{T}$. The reference values of the output current are also chosen as $x_{\text {ref }}=[4,0]^{T}$. Figures $7,8,9$, and 10 show the simulation results. Figure 7 display that the system states are asymptotically convergence to the expected references under the existence of exogenous disturbances and uncertain modeling perturbations. Figures 9 and 10 also indicate that the designed internal models can produce the exogenous disturbances successfully.

\section{Conclusions}

In this paper, a nonlinear backstepping control with multivariable adaptive internal model principle for grid-connected inverter is proposed to reject the harmonic disturbance produced by a class of exosystems under the existence of uncertain items related with system states. Due to the nonlinearity and multiple variables for the original system, a nonlinear and multivariable internal model is constructed. In addition, in order to compensate for the effect of bounded uncertain items, an adaptive control law is designed to realize the realtime estimation of the perturbation. Based on the backstepping control law of the nominal system, a state feedback controller combined with the multivariable internal model and the adaptive control law is designed. A Lyapunov function is constructed and theoretically proves that all the signals of the multivariable closed-loop system are global boundedness. The simulation results show that the proposed control algorithm can guarantee the closed-loop system asymptotically converge to expected references quickly and the designed internal model can produce the exogenous disturbances successfully.

Restricted by the actual problem of grid-connected inverter control under distorted grid voltage, the proposed algorithm in the research aims at rejection of harmonic disturbances, and without considering the nonharmonic disturbances. However, the nonharmonic disturbances may induce adverse impacts, for example, noise and precision reduction. The future research should extend the algorithm to reject the nonharmonic disturbances. 


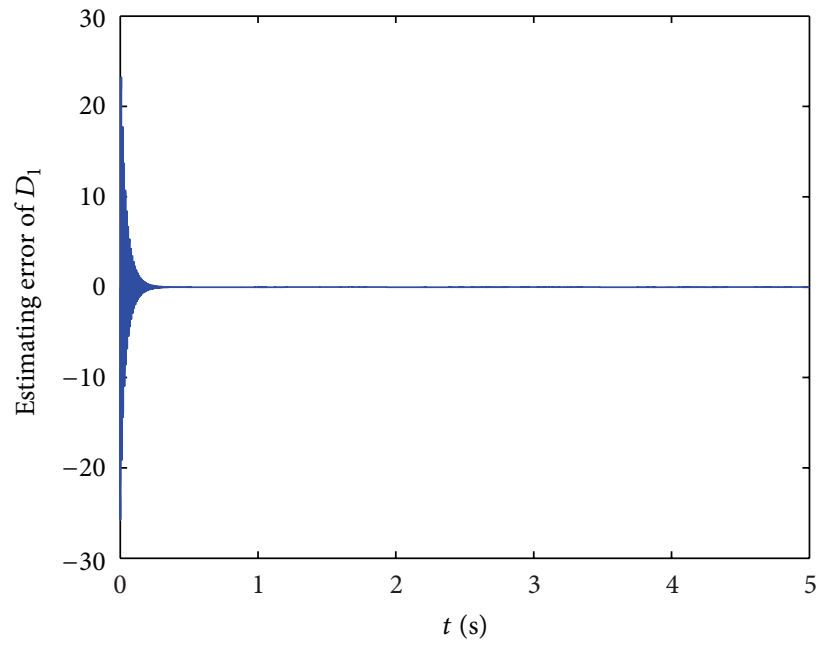

(a)

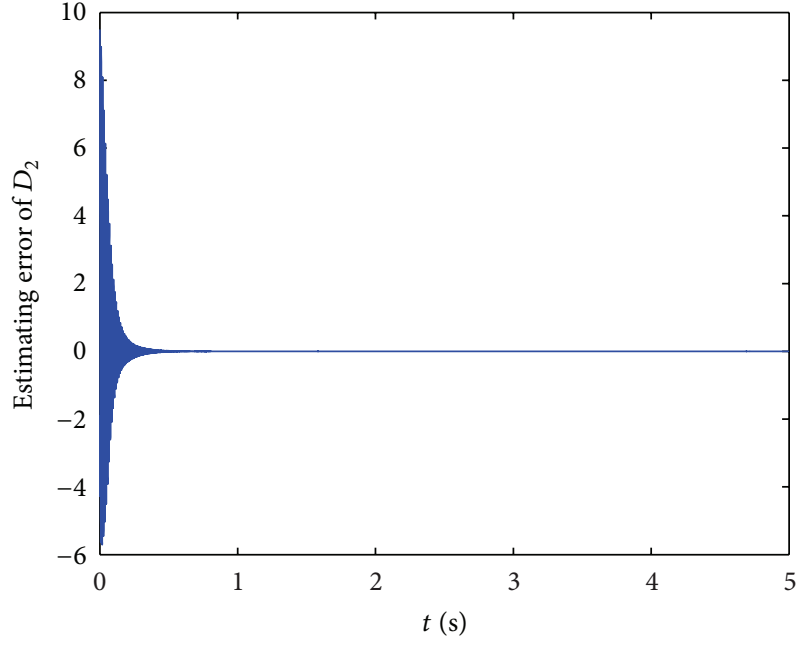

(b)

FIGURE 10: Estimating errors of $D_{1}$ and $D_{2}$.

\section{Acknowledgments}

This research is supported by the Specialized Research Fund for the Doctoral Program of Higher Education of China under Grant no. 20120036130001, the Fundamental Research Funds for the Central Universities of China under Grant no. 11MG40, and the Independent Research Funds of State Key Laboratory of Alternate Electrical Power System with Renewable Energy Sources of China under Grant no. 201209.

\section{References}

[1] H. Lund, "Renewable energy strategies for sustainable development," Energy, vol. 32, no. 6, pp. 912-919, 2007.

[2] T. J. Hammons, "Integrating renewable energy sources into European grids," International Journal of Electrical Power \& Energy Systems, vol. 30, no. 8, pp. 462-475, 2008.

[3] J. López, P. Sanchis, X. Roboam, and L. Marroyo, "Dynamic behavior of the doubly fed induction generator during threephase voltage dips," IEEE Transactions on Energy Conversion, vol. 22, no. 3, pp. 709-717, 2007.

[4] L. Xu and Y. Wang, "Dynamic modeling and control of DFIGbased wind turbines under unbalanced network conditions," IEEE Transactions on Power Systems, vol. 22, no. 1, pp. 314-323, 2007.

[5] L. L. Fan, S. Yuvarajan, and R. Kavasseri, "Harmonic analysis of a DFIG for a wind energy conversion system," IEEE Transactions on Energy Conversion, vol. 25, no. 1, pp. 181-190, 2010.

[6] J. Hu, H. Nian, H. Xu, and Y. He, "Dynamic modeling and improved control of DFIG under distorted grid voltage conditions," IEEE Transactions on Energy Conversion, vol. 26, no. 1, pp. 163-175, 2011.

[7] H. R. Karshenas and H. Saghafi, "Basic criteria in designing LCL filters for grid connected converters," in Proceedings of IEEE International Symposium on Industrial Electronics (ISIE '06), pp. 1996-2000, Montreal, Canada, July 2006.

[8] W. Gullvik, L. Norum, and R. Nilsen, "Active damping of resonance oscillations in LCL-filters based on virtual flux and virtual resistor," in Proceedings of the European Conference on Power Electronics and Applications, pp. 1-10, Aalborg, Denmark, September 2007.

[9] F. Blaabjerg, M. Liserre, P. C. Loh, and R. Teodorescu, "Proportional-resonant controllers and filters for gridconnected voltage-source converters," IEE Proceedings, vol. 153, no. 5, pp. 750-762, 2006.

[10] P. F. Hu, D. Z. Jiang, Y. B. Zhou, J. Guo, and Z. Y. Lin, "Study of the proportional resonant control based modular multilevel converter," in Proceedings of the $3 r d$ International Conference on Digital Manufacturing and Automation, pp. 810-813, Hangzhou, China, 2012.

[11] M. Liserre, R. Teodorescu, and F. Blaabjerg, "Stability of photovoltaic and wind turbine grid-connected inverters for a large set of grid impedance values," IEEE Transactions on Power Electronics, vol. 21, no. 1, pp. 263-272, 2006.

[12] F. Blaabjerg, Z. Chen, and S. B. Kjaer, "Power electronics as efficient interface in dispersed power generation systems," IEEE Transactions on Power Electronics, vol. 19, no. 5, pp. 1184-1194, 2004.

[13] C. Dong, J. M. Zhang, and Z. M. Qian, "An improved repetitive control scheme for grid-connected inverter with frequencyadaptive capability," IEEE Transactions on Industrial Electronics, vol. 60, no. 2, pp. 814-823, 2013.

[14] Y. Y. Tzou, S. L. Jung, and H. C. Yeh, "Adaptive repetitive control of PWM inverters for very low THD AC-voltage regulation with unknown loads," IEEE Transactions on Power Electronics, vol. 14, no. 5, pp. 973-981, 1999.

[15] T. S. Lee, S. J. Chiang, and J. M. Chang, " $H_{\infty}$ loop-shaping controller designs for the single-phase UPS inverters," IEEE Transactions on Power Electronics, vol. 16, no. 4, pp. 473-481, 2001.

[16] G. Weiss, Q. C. Zhong, T. C. Green, and J. Liang, " $H^{\infty}$ repetitive control of DC-AC converters in microgrids," IEEE Transactions on Power Electronics, vol. 19, no. 1, pp. 219-230, 2004.

[17] J. I. Ha, "Current prediction in vector-controlled PWM inverters using single DC-link current sensor," IEEE Transactions on Industrial Electronics, vol. 57, no. 2, pp. 716-726, 2010. 
[18] G. H. Bode, P. C. Loh, M. J. Newman, and D. G. Holmes, "An improved robust predictive current regulation algorithm," IEEE Transactions on Industry Applications, vol. 41, no. 6, pp. 17201733, 2005.

[19] S. Thielemans, T. J. Vyncke, and J. Melkebeek, "Weight factor selection for model-based predictive control of a four-level flying-capacitor inverter," IET Power Electronics, vol. 5, no. 3, pp. 323-333, 2012.

[20] T. Wang, S. C. Tong, and Y. M. Li, "Robust adaptive fuzzy control for nonlinear system with dynamic uncertainties based on backstepping," International Journal of Innovative Computing, Information and Control, vol. 5, no. 9, pp. 2675-2688, 2009.

[21] S. C. Tong, Y. M. Li, and P. Shi, "Fuzzy adaptive backstepping robust control for SISO nonlinear system with dynamic uncertainties," Information Sciences, vol. 179, no. 9, pp. 1319-1332, 2009.

[22] F. Zouari, K. S. Ben, and M. Benrejeb, "Robust adaptive control for a class of nonlinear systems using the backstepping method," International Journal of Advanced Robotic Systems, vol. 10, pp. 112,2013

[23] Y. J. Liu, W. Wang, S. C. Tong, and Y. S. Liu, "Robust adaptive tracking control for nonlinear systems based on bounds of fuzzy approximation parameters," IEEE Transactions on Systems, Man, and Cybernetics A, vol. 40, no. 1, pp. 170-184, 2010.

[24] R. Ketata, Y. Rezgui, and N. Derbel, "Stability and robustness of fuzzy adaptive control of nonlinear systems," Applied Soft Computing, vol. 11, no. 1, pp. 166-178, 2011.

[25] S. Tong, C. Liu, and Y. Li, "Fuzzy-adaptive decentralized output-feedback control for large-scale nonlinear systems with dynamical uncertainties," IEEE Transactions on Fuzzy Systems, vol. 18, no. 5, pp. 845-861, 2010.

[26] Y. J. Liu and W. Wang, "Adaptive fuzzy control for a class of uncertain nonaffine nonlinear systems," Information Sciences, vol. 177, no. 18, pp. 3901-3917, 2007.

[27] S. T. Liu, Y. Jiang, and P. Liu, "Rejection of nonharmonic disturbances in nonlinear systems," Kybernetika, vol. 46, no. 5, pp. 785-798, 2010.

[28] C. L. Chen, Z. T. Ding, and B. Lennox, "Rejection of nonharmonic disturbances in nonlinear systems with semi-global stability," IEEE Transactions on Circuit System, vol. 55, no. 12, pp. 1289-1293, 2008.

[29] Z. W. Ping and J. Huang, "Global robust output regulation for a class of multivariable systems and its application to a motor drive system," in Proceedings of the American Control Conference (ACC '11), pp. 4560-4565, San Francisco, Calif, USA, June-July 2011.

[30] H. K. Khalil, Nonlinear Systems, Prentice-Hall, New Jersey, NJ, USA, 3rd edition, 2002.

[31] A. Isidori, Nonlinear Control Systems, Springer, New York, NY, USA, 3rd edition, 1995.

[32] M. M. Polycarpou and P. A. Ioannou, "A robust adaptive nonlinear control design," Automatica, vol. 32, no. 3, pp. 423427, 1996. 


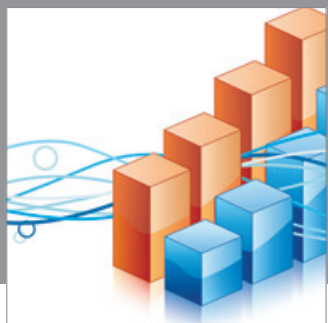

Advances in

Operations Research

mansans

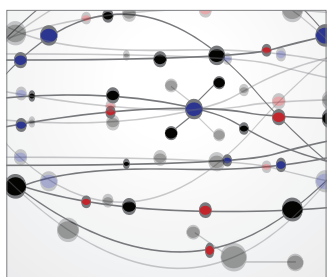

The Scientific World Journal
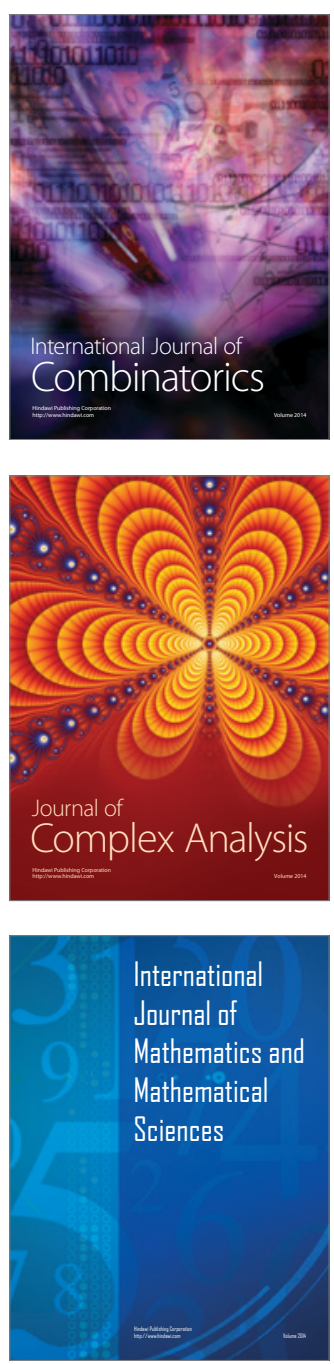
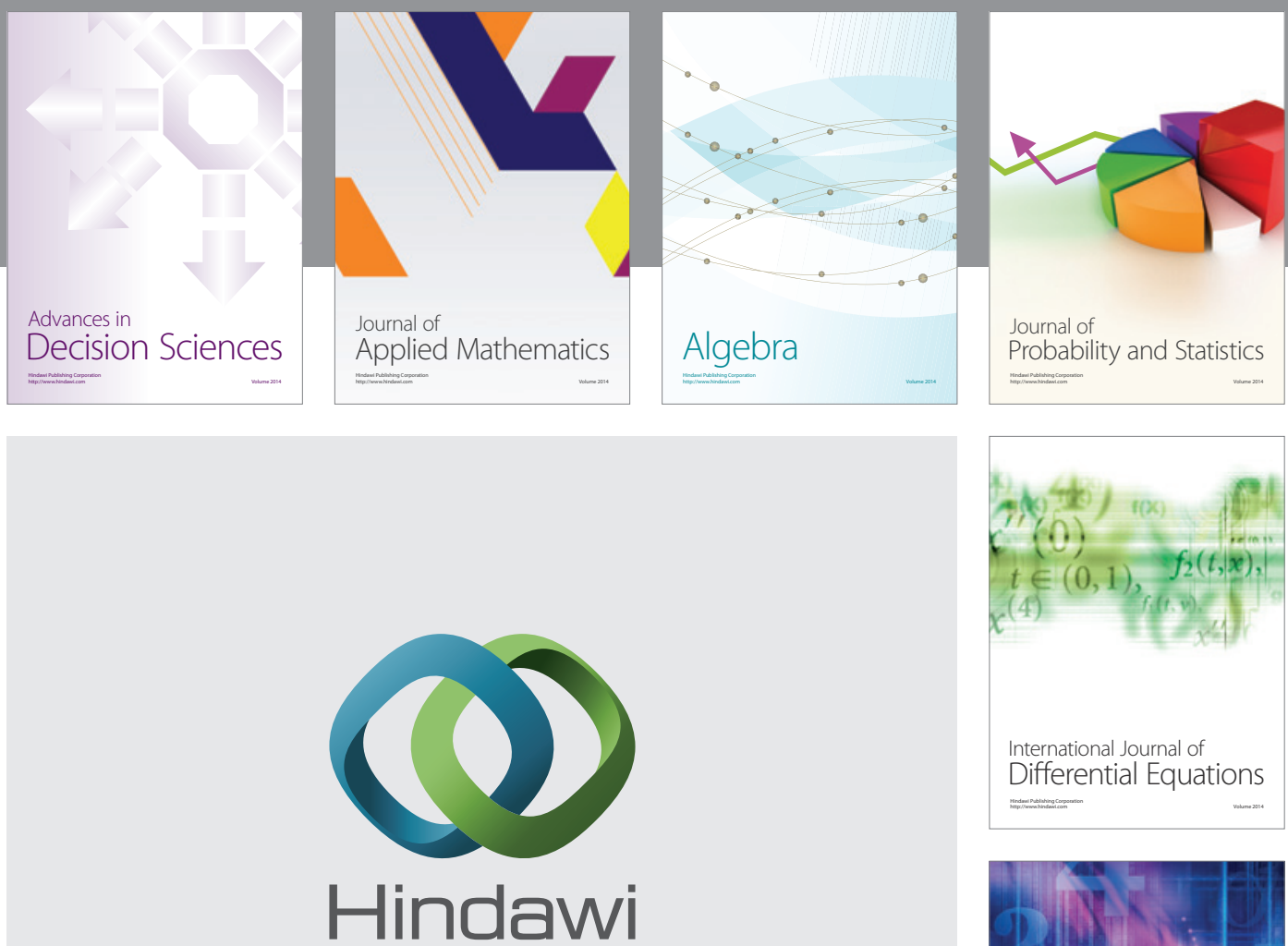

Submit your manuscripts at http://www.hindawi.com
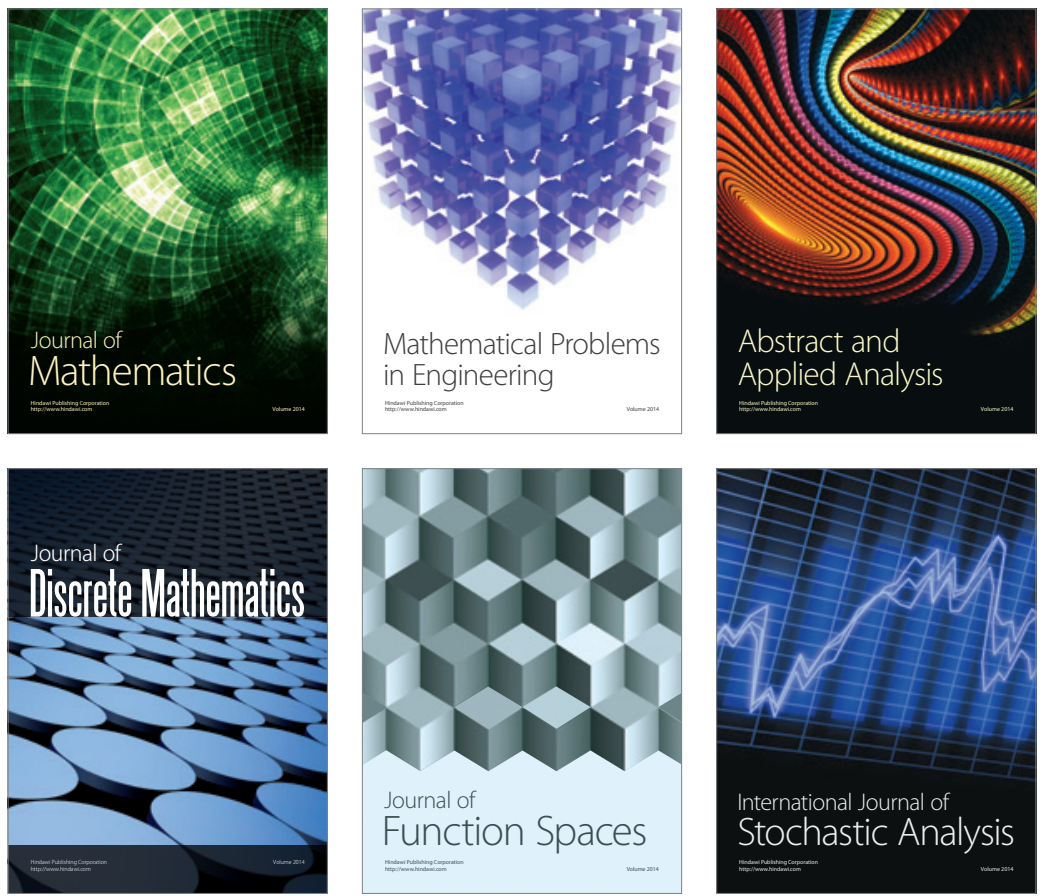

Journal of

Function Spaces

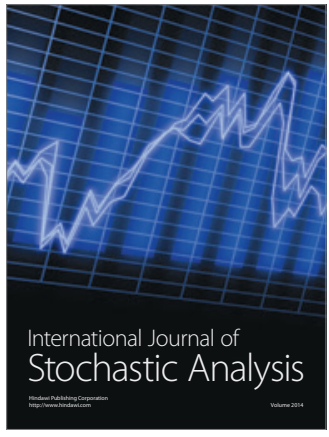

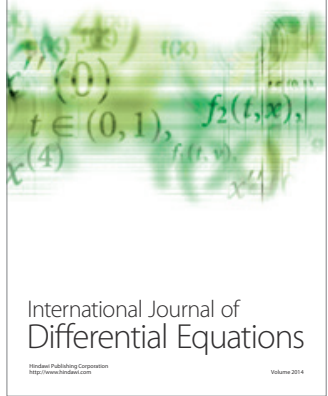
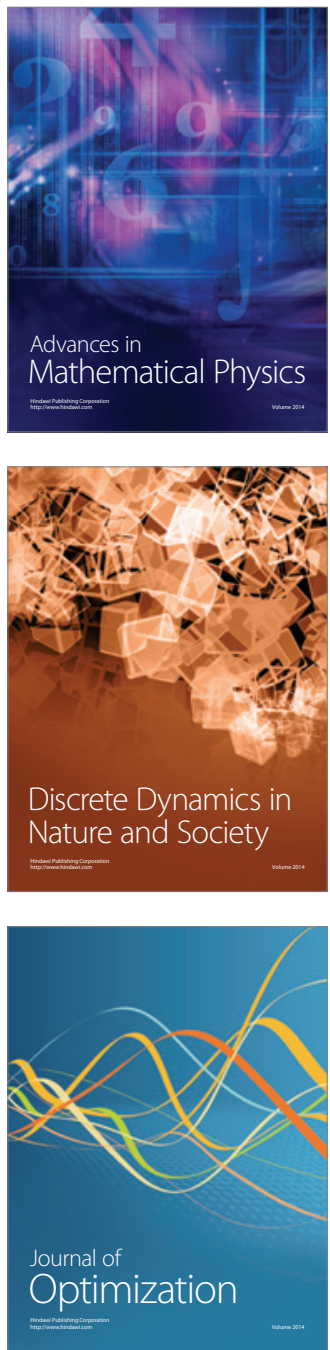